\title{
LMS-Verify: Abstraction without Regret for Verified Systems Programming
}

\author{
Nada Amin* Tiark Rompf ${ }^{\dagger}$ \\ ${ }^{*}$ EPFL: \{first.last\}@epfl.ch \\ ${ }^{\dagger}$ Purdue University: \{first $\}$ @purdue.edu
}

\begin{abstract}
Performance critical software is almost always developed in C, as programmers do not trust high-level languages to deliver the same reliable performance. This is bad because low-level code in unsafe languages attracts security vulnerabilities and because development is far less productive, with PL advances mostly lost on programmers operating under tight performance constraints. Highlevel languages provide memory safety out of the box, but they are deemed too slow and unpredictable for serious system software.

Recent years have seen a surge in staging and generative programming: the key idea is to use high-level languages and their abstraction power as glorified macro systems to compose code fragments in first-order, potentially domain-specific, intermediate languages, from which fast $\mathrm{C}$ can be emitted. But what about security? Since the end result is still $\mathrm{C}$ code, the safety guarantees of the high-level host language are lost.

In this paper, we extend this generative approach to emit ACSL specifications along with $\mathrm{C}$ code. We demonstrate that staging achieves "abstraction without regret" for verification: we show how high-level programming models, in particular higher-order composable contracts from dynamic languages, can be used at generation time to compose and generate first-order specifications that can be statically checked by existing tools. We also show how type classes can automatically attach invariants to data types, reducing the need for repetitive manual annotations.

We evaluate our system on several case studies that varyingly exercise verification of memory safety, overflow safety, and functional correctness. We feature an HTTP parser that is (1) fast (2) high-level: implemented using staged parser combinators (3) secure: with verified memory safety. This result is significant, as input parsing is a key attack vector, and vulnerabilities related to HTTP parsing have been documented in all widely-used web servers.
\end{abstract}

Categories and Subject Descriptors $\quad$ D.3.3 [Programming Languages]: Language Constructs and Features

Keywords contracts, blame, memory safety, Frama-C, LMS, DSLs, verification, security

Permission to make digital or hard copies of all or part of this work for personal or classroom use is granted without fee provided that copies are not made or distributed for profit or commercial advantage and that copies bear this notice and the full citation on the first page. Copyrights for components of this work owned by others than ACM must be honored. Abstracting with credit is permitted. To copy otherwise, or republish, to post on servers or to redistribute to lists, requires prior specific permission and/or a fee. Request permissions from Permissions@ acm.org.

Copyright is held by the owner/author(s). Publication rights licensed to ACM.

POPL'17, January 15-21, 2017, Paris, France

ACM. 978-1-4503-4660-3/17/01 ...\$15.00

http://dx.doi.org/10.1145/3009837.3009867

\section{Introduction}

How should we build systems that are performant, secure, and correct? Today, systems-level software such as network stacks, databases, and control code in embedded devices is almost always developed in C. This is bad, for at least two reasons. First, development is far less agile and productive than in higher level languages, and second, low-level code in unsafe languages invites security vulnerabilities.

High-level languages, on the other hand, rule out entire classes of vulnerabilities through built-in memory safety guarantees, and many of them provide elaborate mechanisms for programming with contracts, which enforce user-defined specifications at runtime. But while implementations of high-level languages have come a long way, programmers cannot trust them to deliver the same reliable performance as handwritten $\mathrm{C}$ code. Contract monitoring at runtime, in particular, can have prohibitive overhead [80, 87]. As a consequence, PL advances are mostly lost on programmers operating under tight performance constraints. Sound static verification of general-purpose $\mathrm{C}$ code is possible, but has an extraordinary cost in terms of user annotations, and is thus rarely done in practice. But tools are advancing fast, and formal verification is on the verge of becoming practical for larger classes of software.

Motivated by the apparent trade-off between productivity and performance, researchers have argued [71] for a rethinking of the role of high-level languages in performance critical code, with the goal of allowing developers to leverage high-level programming abstractions without the hefty price in performance. The shift in perspective that enables this vision of "abstraction without regret" is a properly executed form of generative programming: instead of running the whole system in a high-level managed language runtime, the abstraction power of high-level languages can be focused on generating and composing pieces of low-level code.

Generative programming has proven successful for numerical kernels such as FFTs [61], for DSLs in big-data processing [13], but also in domains like database query engines [47], and parsers for communication protocols [43]. In these traditional strongholds of low-level programming, generative programming has largely lived up to its promise: programmers reap the benefits of programming in a high-level language without the low-level language drawbacks.

However, previous work has only looked at productivity benefits, not at security and correctness. Since low-level code is still being generated, the safety guarantees usually associated with highlevel languages (e.g. memory safety) do not carry over. And what about functional correctness? We have to trust the high-level code and the generator, either or both of which might contain bugs.

The main contribution of this paper is to show that "abstraction without regret" holds for security and verification as it does for performance. We demonstrate how generative programming frame- 
works can be extended to emit low-level code that can be formally verified using existing C-level verification tools. For this, we provide a contract API to specify user-defined assertions as well as pre- and post-conditions. Since we are working in a metaprogramming environment, this facility effectively enables programming with higher-order composable contracts at generation time. But unlike contract systems in dynamic languages, our contracts result in first-order assertions in the generated code. These can be statically checked and never lead to any runtime overhead.

While verification tools for $\mathrm{C}$ have come a long way, they still require an inordinate amount of annotations to verify even small programs. Fortunately we can capitalize on the fact that we are constructing program pieces from within a meta-language again. By not generating target code directly but going through one or more domain-specific intermediate languages, we can restrict the programming model and include e.g. effect and aliasing information in the IR. From such an enriched IR, we show that we can generate a large subset of the required annotations such as loop invariants and separation properties automatically. This automation proves vital in reducing the annotation burden for the programmer.

Finally, generative programming does not need to be an all-ornothing approach. It is easy to specify boundary APIs and generate only critical parts of a system, while other parts are written by hand. This is a key reason why it is attractive to target standardized specification languages such as ACSL (ANSI/ISO C Specification Language) [7].

In short, we show that generative programming is useful not just for performance, but also for verification. In our approach, we generate not just low-level code (e.g. C), but also low-level specs (e.g. ACSL annotations), so that the output can be verified (e.g. to be memory safe) by an independent tool (e.g. Frama-C). We make the following contributions:

- We review how generative programming enables "abstraction without regret" for high-performance and low-level code, stressing the key insights: (1) we can use abstractions such as higher-order functions, objects, type classes, etc. at generation time without incurring any cost at runtime, (2) we can use the meta-language type system to enforce certain desired restrictions on the generated code, in particular that functions and mutable variables behave in a second-class way (Section 2).

- We extend the programming model with higher-order, composable, contracts. Since we generate only top-level functions with first-order code, i.e. inlined or named function applications, we are able to (1) statically check the contracts in the low-level code, including the higher-order pieces, and (2) reliably assign "blame" in this generative setting (Section 3).

- We apply generative programming to compose not just code fragments, but also contracts and specifications. We show how to further alleviate the annotation burden by (1) associating invariants to data types via type classes, (2) generating both code and specification logic from the same high-level source, (3) inferring loop and separation properties from the IR, and (4) through custom domain-specific loop constructs (Section 4).

- We evaluate our system on several case studies that exercise memory safety, overflow safety, and functional correctness, including an HTTP parser that is (1) fast, (2) high-level: implemented using staged parser combinators, (3) secure: with verified memory safety. This result is significant, as input parsing is a key attack vector, and vulnerabilities related to HTTP parsing have been found in all widely-used web servers (Section 5).

Section 6 provides a broader perspective, and Section 7 surveys related work. Our evaluation suggests that the usual patterns of generative programming (e.g. generic instantiation, turning an interpreter into a compiler) scale surprisingly well to verification.

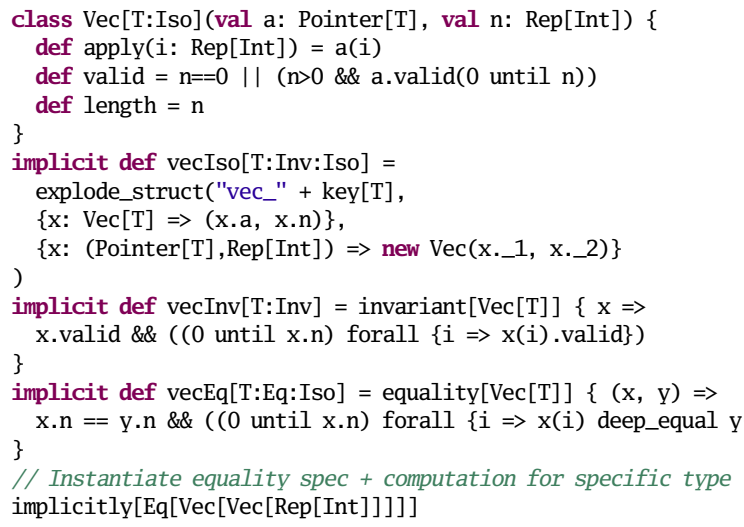

Figure 1. Higher-order generic source in Scala, generating lowlevel annotated code of Figure 2.

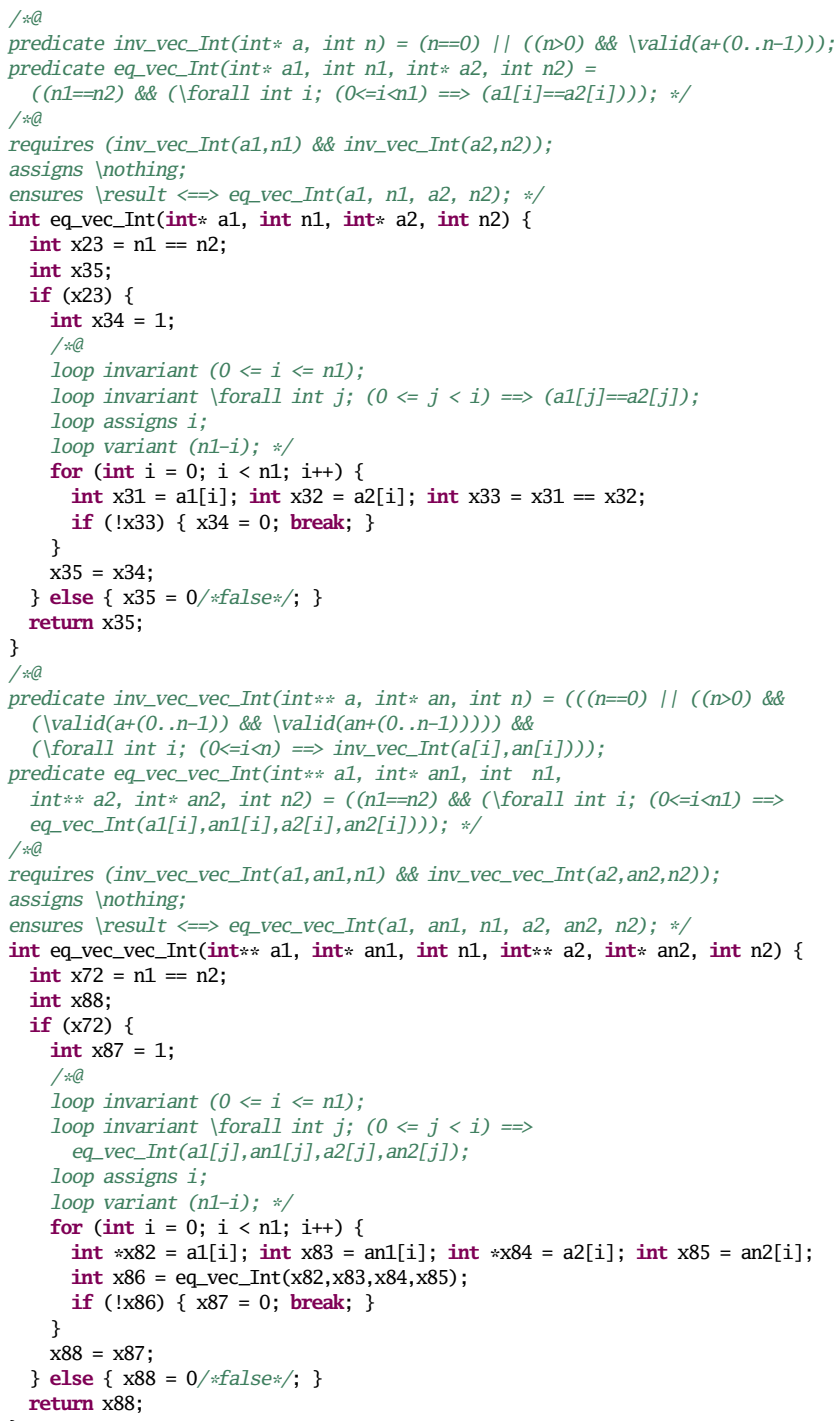

Figure 2. Emitted low-level first-order code in $\mathrm{C}$ with specifications in ACSL, generated from high-level generic source of Figure 1 , modulo some local variable renaming for clarity. 


\begin{tabular}{l}
\hline vector of integers \\
int* element array \\
int length \\
vector of vector of integers \\
int** element array by outer then inner index \\
int* array for inner length by outer index \\
int length (of outer)
\end{tabular}

Figure 3. Representation of vectors in low-level code of Figure 2. Choice here explodes source fields (item pointer, length) into variables, and flattens nested structures akin to "struct of arrays".

As a teaser, consider the source and target code shown in Figures 1 and 2, respectively (it is not necessary to understand it in detail yet; many parts of it will be explained in Section 2). Based on a generic definition of a Vec class, we define generic type classes that define data layout, data invariants, and equality predicates. When we instantiate these generic templates to a concrete type, we will emit the corresponding specialized first-order C code - complete with ACSL specifications. Here in the generator, we ask for the equality instance for a vector of vectors of integers. In the generated code, we get the data invariants (as ACSL logic) and the equality predicates (as both C code and ACSL logic), for vector of integers and vector of vectors of integers, where the latter uses the former internally. The specifications gracefully handle complex internal data layouts: In the low-level code, a vector of integers is represented by two separate $\mathrm{C}$ values (element data and length), and a vector of vectors of integers by three, as outlined in Figure 3.

\section{High-Level Low-Level Programming}

How can we do low-level programming in a high-level language? The key idea of generative programming is to use the high-level language as a glorified macro system to compose fragments of lowlevel code in a (potentially domain-specific) intermediate language (IR). From this IR, low-level efficient $\mathrm{C}$ code can be emitted, possibly after further optimizations.

C as an Embedded DSL in Scala We base our development on Scala and LMS (Lightweight Modular Staging) [72]. The main idea of LMS is to provide a type-based embedding of the IR into the host language (Scala). A special type constructor Rep[T] is used to mark staged expression of type T. In other words, a value of type Rep[Int] or Rep[String] denotes a representation of an Int or String value in generated code.

Scala dialects provide flexible forms of overloading that extend to language built-ins like conditionals [70]. An expression such as

if $(a<b)$ a else $b$

Will be desugared into method calls:

_ifThenElse(a.<(b), a, b)

And with LMS, two overloaded versions exist:

def _ifThenElse[T] (c: Boolean, a: $\Rightarrow \mathrm{T}, \mathrm{b}: \Rightarrow \mathrm{T}$ ): T

def _ifThenElse[T] (c: Rep[Boolean], a: $\Rightarrow \operatorname{Rep}[T], b: \Rightarrow \operatorname{Rep}[T]): \operatorname{Rep}[T]$ )

The Scala compiler's local type inference essentially performs a local binding-time analysis to determine which parts of an expression are evaluated right away and which parts are staged, becoming part of the generated IR.

To continue our example, if either a or $b$ are Rep[Int] values, $\mathrm{a}<\mathrm{b}$ will have type Rep[Boolean]. In this case a conditional will be generated. If $a$ and $b$ are of type Int, expression $a<b$ will be of type Boolean and one of the conditional branches will be picked at staging (i.e. IR generation) time.

In traditional multi-stage programming approaches, staging is often homogeneous: the host language and the target language of code generation is the same. Here, we are mostly interested in generating $\mathrm{C}$ code from Scala, so we are dealing with a heterogeneous embedding. This gives us some freedom to provide types that have no direct meta-language equivalent. For example, for low-level systems programming we might need to deal with pointers, and add a corresponding Pointer[T] type to our embedding. In the same vein, we can add facilities like malloc and free for manual memory management, which do not have an equivalent in Scala.

In our setting, the target language is more restricted than the meta-language, as $\mathrm{C}$ does not provide features like closures, pattern matching, or managed memory. For this reason, and because analysis and verification of higher-order constructs is fundamentally more difficult, we want to generate mostly first-order entities.

Our IR is designed to be unparsed to $\mathrm{C}$ almost directly. Compared to $\mathrm{C}$, we remove function pointers, and the ability to take the address of a value $x$ via \&x. These restrictions enable us to maintain some useful separation properties in the IR by design. We add support for nested functions, which requires a lambda lifting transform before unparsing (see Section 2.3).

\subsection{Deep Linguistic Reuse}

Working in a generative setting, the first key insight is that we can use arbitrary facilities of the meta-language at generation time, without any overhead in the generated code.

Classes and Objects On top of the primitives provided by the IR, we can, e.g., define a data structure for vectors, using high-level object-oriented abstractions in the meta-language for compositionality, similar to what we did in Figure 1:

$$
\begin{aligned}
& \text { class Vec[T] (val a: Rep[Pointer[T]], val } n: \operatorname{Rep}[\operatorname{Int}])\{ \\
& \text { def apply(i: Rep[Int]) }=\mathrm{a}(\mathrm{i}) ; \text { def length }=\mathrm{n}\}
\end{aligned}
$$

In the generated code, all the abstraction overhead of classes and methods will be stripped away, leaving just the low-level pointer manipulations.

Shallow Functions We have seen primitive operations like $<$ and conditionals above. But how can we effectively work with functions in generative programming? We first note that we can transparently use meta-language functions for composition:

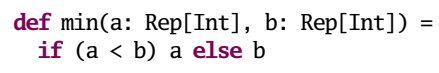

In this case, function min is a meta-language, i.e. staging-time, function of type $\operatorname{Rep}[A]=>\operatorname{Rep}[B]$. Such a function will be inlined when called, without leaving a trace of the function abstraction in the generated code.

Since such functions are purely staging-time abstractions, we can freely define higher-order functions, too, for example to traverse a Vec, and to compute aggregates:

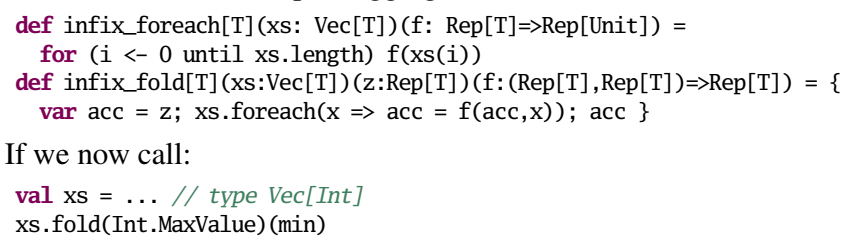

Then we will generate the following $C$ code:

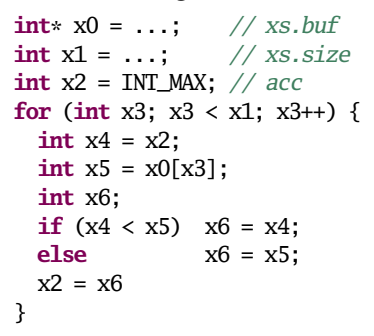

As we can see, the generated code is completely first-order. There are no functions present, and much less higher-order ones. 
Generic Programming with Type Classes Most likely, we will reach a point where we would like to extend the functionality of Vec objects further. Perhaps we would like to compare two Vecs for equality. This requires that the elements of a Vec are comparable, too: a prime example of generic programming, which we can solve elegantly using type classes [90].

We begin by defining the type class signature, with a Rep[Boolean] result for the comparison:

trait Eq[T] \{ def eq(a: T, b: T): Rep[Boolean] \}

And we provide a helper method to construct instances from a given comparison function:

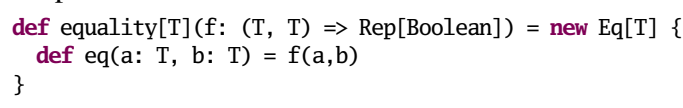

We also add an implicit enrichment

implicit class EqOps[T:Eq] (a: T) \{ \}

def deep_equal(b: T): Rep[Boolean] = implicitly[Eq[T] $]. e q(a, b)$

which enables us to call a deep_equal $b$ when $a$ and $b$ are objects of a type $\mathrm{T}$, for which a type class instance $\mathrm{Eq}[\mathrm{T}]$ exists.

To instantiate equality for Vecs, we need to create the corresponding Eq instance and make it available as implicit. Note the use of deep_equal to compare the elements of the Vec:

implicit def vecEq[T:Eq] = equality[Vec[T] $\{(\mathrm{x}, \mathrm{y}) \Rightarrow$

$\mathrm{x} \cdot \mathrm{n}==\mathrm{y} \cdot \mathrm{n} \& \&((0$ until $\mathrm{x} . \mathrm{n})$ forall $\{\mathrm{i} \Rightarrow \mathrm{x}(\mathrm{i})$ deep_equal $\mathrm{y}(\mathrm{i})\})\}$

Interestingly, type classes can be used without any modification in a generative setting. The key insight is that type classes come with a natural stage distinction, given by the decoupling of data (which, in our case is staged) and behaviour (executed at staging time). Thus, we can reuse the host language once more and get this powerful pattern for free in staged code.

\subsection{First-Class Now, Second-Class Later}

The second key insight is that, through clever programming patterns, we can leverage the meta-language type system to ensure key desirable restrictions on generated code.

Higher-order programming and first-class entities are fundamentally linked. In general, language features are said to be firstclass if they can be used without restrictions [78], and second-class if they can be used only in certain ways but not others. For example, second-class functions can only be called, but not stored in mutable variables or returned from other functions. Second-class mutable variables can be read and written, but cannot be stored in other variables. On the flip side, first-order code can use only secondclass patterns while higher-order code uses first-class patterns. For example, first-order functions can use only second-class functions, while higher-order functions use first-class functions.

An extremely useful design pattern in generative programming is that second-class constructs from the target language may be abstracted over and used as first-class objects at generation time. First-class objects in generated code must be of type Rep[T], but we are free to provide additional abstractions that use Rep types internally but make them accessible only through a purely secondclass API. We will see several examples below.

Deep Functions Above, we have discussed shallow functions, which only exist at staging time, and can be effectively used as macros, to achieve the effect of inlining. But of course inlining is not always the desired choice. Sometimes we want to generate actual function definitions. A truly first-class function would have type $\operatorname{Rep}[A=>B]$. But since there is no $C$ equivalent, it would not make much sense to support such abstractions. Instead, we introduce an operator fundef that creates a function definition, but its return type is again $\operatorname{Rep}[\mathrm{A}]=>\operatorname{Rep}[\mathrm{B}]$. This means that the defined function can only be called, but it cannot be referenced as a first-

class value in the generated code. Thus, fundef is an abstraction to create second-class functions, with restricted usage patterns.

If we change the code above to use fundef:

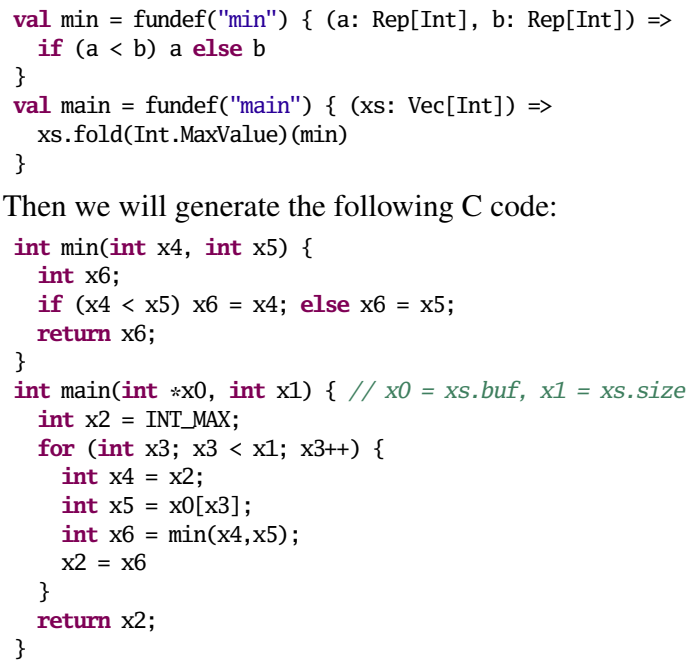

Then we will generate the following $\mathrm{C}$ code:

Mutable Variables and Buffers Similar considerations as with functions apply when working with mutable state. In many cases we want to work with local variables in the generated code, but treat them also as first-class staging-time objects. The type of variables we use is plain $\operatorname{Var}[\mathrm{T}]$, i.e. not a Rep type. But read and update methods on Var objects take and return Rep types, providing a second-class staging-time abstraction over generated code.

Nevertheless, we can treat variables as first-class entities while generating code. In particular, we can emit a flexible amount of local variables, based on some input data. For example,

$\operatorname{List}(1,2,3) \cdot \operatorname{map}(\mathrm{x}=>$ var_new(x)) //: List [Var[Int]]

generates code:

int $\mathrm{x} 1=1$; int $\mathrm{x} 2=2$; int $\mathrm{x} 3=3$;

Or we can define staging-time functions that take variables as arguments, enabling programming patterns similar to ML-style firstclass references:

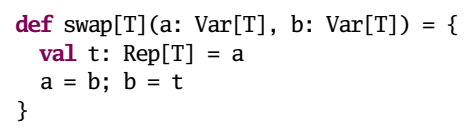

The key difference to first-class references however is that their second-class nature bounds the lifetime of variables in the generated code. As fundef's type signature does not permit returning a $\operatorname{Var}[\mathrm{T}]$ value, its lifetime cannot exceed its meta-language scope.

We can generalize from individual variables to second-class Buffer[T] objects, which leads to a simple but effective form of region-based memory policy.

Controlling Object Representation with Type Classes Consider again our Vec example. Right now Vec objects are second-class, like variables, so they cannot be arguments or return values of deep functions. This is rather limiting, and we might also want to nest Vecs, but we cannot: due to their second-class status, we can only put Rep values into Vecs.

To make Vecs first-class, type classes come to the rescue again. Based on ideas from polytypic programming $[41,83,77]$ we introduce a type class Iso[T], which defines a mapping between Vec and the Rep types it consists of:

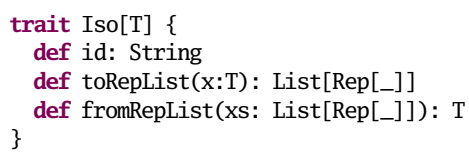


We create the corresponding instance for Vec as follows:

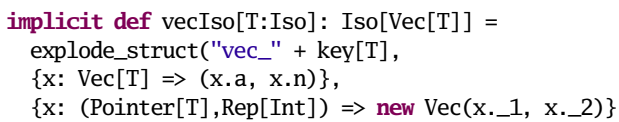

To go one step further and allow nesting of Vec objects, we need to generalize our low-level representation of pointers from Rep[Pointer[T]] to Pointer[T], with a suitable Iso instance for Pointer[T]. There are different options how such nested data can be stored. The most common variant would be as array of structs (AoS), but for performance, it is often desired to transpose the layout and store data as struct of arrays (SoA). The Iso implementation can pick either, but using explode_struct we settle for SoA as illustrated in Figure 3, in the code shown in Figure 2.

The internal definition of fundef requires an Iso instance for the argument and return type, in order to generate the proper function signatures. A first approximation of its signature would be:

def fundef[A:Iso,B:Iso](f: $A \Rightarrow B$ ): A $\Rightarrow B$

However we can take this pattern further and introduce a distinction between argument and return types. This is useful to ensure a "downward funarg" policy for certain types like stack-allocated buffers, which guarantees that object created inside a function cannot escape this scope. Thus, the actual signature of fundef is:

def fundef[A:DemiIso,B:Iso](f: A $\Rightarrow$ B): A $\Rightarrow$ B

Where DemiIso has the same functionality as Iso but serves as a marker for types like Var or Buffer that can only appear in function argument but not in return position. We treat Iso as strictly more general than DemiIso by defining an implicit conversion from Iso to DemiIso, but for types like Var or Buffer we only implement DemiIso, not Iso. This guarantees the desired behavior with respect to stackbounded object lifetimes.

To wrap up this section, we highlight the insight that with type classes, we can control the shape of data (as in normal programming) but also the shape of generated code. In particular we can enable or restrict values of specific types to be nested, or to appear in function argument or return types.

\subsection{IR Transformations and Unparsing to $\mathrm{C}$}

At the IR level, we can implement various analyses and optimizations before we generate target code. Unparsing to $\mathrm{C}$ is straightforward, since the IR is already low-level and designed to map to $C$ in a direct way. One aspect still requires further attention:

Lambda Lifting Since Scala supports nested functions, invocations of fundef may also be nested, and refer to objects, including Vars, from enclosing scopes:

$\operatorname{var} \mathrm{a}=\ldots / / \operatorname{Var}[\operatorname{Int}]$

val $\mathrm{f}=$ fundef $\{\mathrm{x}$ : Rep[Int $] \Rightarrow \mathrm{a}+=\mathrm{x}\}$

$\mathrm{f}(2)$

Of course, $\mathrm{C}$ does not support nested functions, so we have to apply a lambda lifting transformation on the IR level before emitting C. This lambda lifting pass takes variables into account and supports non-local variables by passing pointers. For the input above, the generated code will look (roughly) like this, with a pointer to variable a added to function $\mathrm{f}$ 's argument list.

int $\mathrm{f}($ int $* \mathrm{a}$, int $\mathrm{x})\{* \mathrm{a}+=\mathrm{x} ;\}$

int $\mathrm{a}=\ldots$

$\mathrm{f}(\& \mathrm{a}, 2)$

Passing a pointer to variable a to function $f$ is safe, because the variable can never escape its defining scope, and because we do not support function pointers as first-class entities. Similarly, variables cannot be stored in other variables, and they cannot be returned from functions. Together, these constraints maintain our stackbounded lifetime guarantees for second-class values.

\subsection{Problems}

All in all, generative programming provides a valuable approach to raise the level of abstraction in low-level systems development. Programmers obtain some nice high-level guarantees, including type safety, and certain properties about scoping, for example that variables and pointers do not escape. But how reliable are those guarantees? Since we are still generating low-level C code, we have to take into account bugs and inconsistencies at all levels of the stack.

First, even though we are working in a safe high-level language, we are giving up built-in properties like memory safety the very moment we decide to generate $\mathrm{C}$ code. We are no longer protected against out-of-bounds memory accesses, buffer overflows, or other kinds of errors that are all too often the source of security vulnerabilities. Of course it would be possible to generate appropriate checks, but the associated performance overhead would most likely not be acceptable.

Second, we cannot trust the generator to do the right thing, especially if one or more levels of IRs are involved in the generation. A concrete example we have come to know about was the data loading code in a database query engine built using LMS. While the data field abstraction was properly implemented to work with fixed-length strings, programmers had not accounted for the additional NULL terminator that is present in C strings, but not in the high-level abstraction.

Third, we do not know if the program itself does the right thing, since we have no principled way of incorporating verification of functional correctness properties into our program generators, except perhaps by generating costly runtime checks.

The central thrust of this paper is to show that these shortcomings can be fixed in an elegant and principled way, by generating $\mathrm{C}$ code along with specifications that can be automatically verified by independent tools. Our development uses Frama-C [21] and the ANSI C Specification Language (ACSL) [7] but other tools like VeriFast [39] would be viable alternatives.

\section{Contracts}

Specifications and in particular function contracts play a key role in program verification. The key idea of LMS-Verify, our generative programming system geared towards verifiable code, is to not only generate straight executable $\mathrm{C}$ code, but generate contracts and assertions alongside. These could be checked dynamically, but we seek full static verification in order to eliminate all possible runtime overhead. To achieve static verification, we need to generate enough assertions, loop invariants, and function pre- and postconditions so that existing verification tools are able to reason about every possible behavior of a certain piece of generated code.

Contracts for Deep Functions In their simplest model, function contracts consist of pre- and post-conditions for a given function definition. We can add corresponding functionality to fundef:

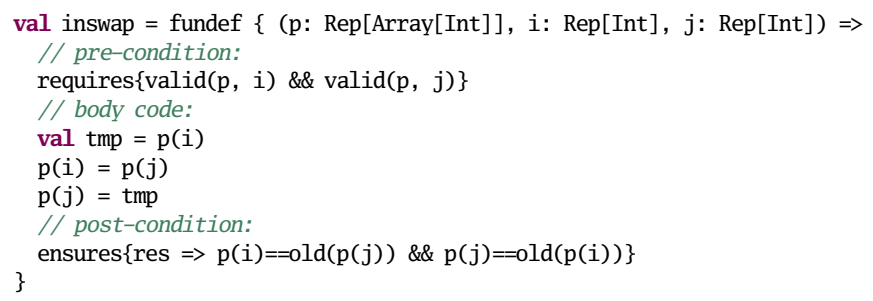

The pre- and post-conditions are just regular staged expressions (type Rep[Boolean]), but since they occur in specification context they will not generate executable C code but ACSL annotations: 


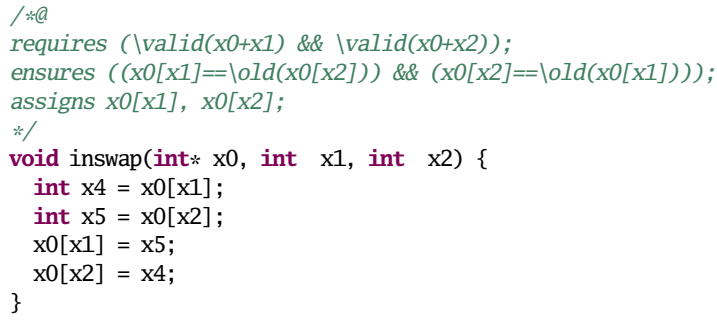

While in general most kinds of expressions can either generate $\mathrm{C}$ or ACSL code there are certain exceptions: side-effecting statements can only generate $\mathrm{C}$ code, and some expressions like old and valid are only available in specifications. Using such an expression in an incompatible context will result in an error at staging time.

Shallow Contracts Some dynamic languages such as Racket [29] offer elaborate APIs for higher-order composable contracts [30]. It turns out that we can use the same pattern we used for working with higher-order functions at generation time again for contracts: we use meta-language abstractions to compose computations that will produce the right assertions in the generated code.

In essence, adding pre- and post-conditions to a function just corresponds to eta-expansion, inserting an appropriate check before or after the function is executed. However, just a simple check is not enough. Since pre- and post-conditions form a contract between caller and callee of a functions, we would like to make sure that we can blame the right party for any failure.

Blame Assignment To assign blame for contract violations, we use Scala's implicit SourceContext facility [70]. Whenever the Scala compiler encounters a method call that requires a SourceContext argument maked as implicit, the compiler will synthesize such an object and initialize it with the source file and line number at the method call site.

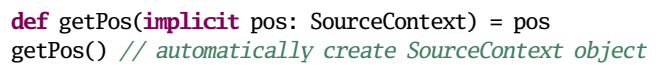

We can use this approach to define our own class of function objects FuncShallow that will store the source location of their definition as well as obtain the caller's source location when invoked.

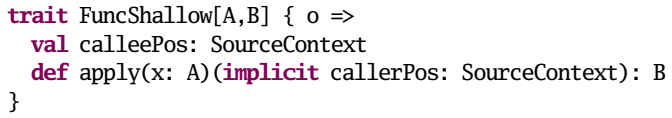

Base on this function class, we add contracts via pre- and postconditions.

Contracts are added by eta-expanding and guarding pre/post conditions with an assert, blaming callerPos or calleePos respectively for any contract violations that might happen.

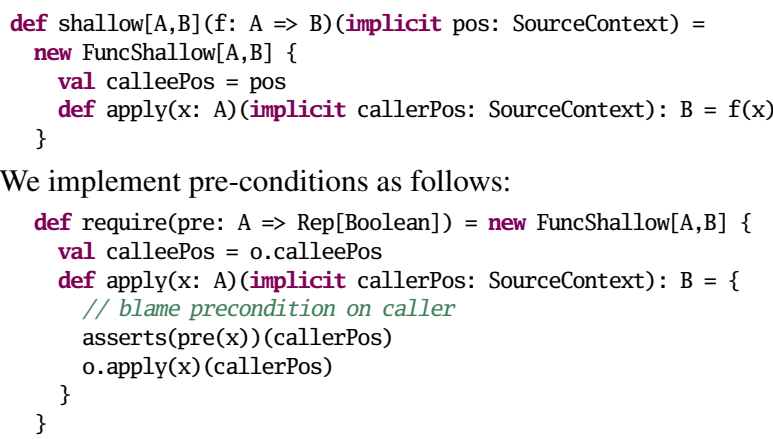

Post-conditions using ensures are analogous to the implementation for pre-conditions but they blame the callee instead of the caller. Note that the contract implementations use an asserts statement that looks very similar to a normal runtime check, but instead it will generate an ACSL assertion in the output $\mathrm{C}$ file. For blame assignment, we emit source information in the generated code using \#line file directives (more below).

Higher-Order Contracts Straightforward contracts have their limits, even though we can make them tremendously more useful by being able to generate assertions instead of writing them all by hand. A key limitation is that we cannot easily state properties about functions that are passed as arguments or returned from other functions.

The idea of higher-order contracts is to use eta-expansion again, and insert code before and after the function, which will inspect its arguments and results. In the implementation, nested contracts are linked to their parents, and the blame assignment flips between caller and callee whenever we enter a pre-condition, i.e. increase the depth to the left of a function arrow. This approach follows exactly the standard algorithm for blame assignment in dynamically monitored contracts [30].

Here is an example, taken from [59], that transforms a function from even to even numbers into a function from odd to odd numbers:

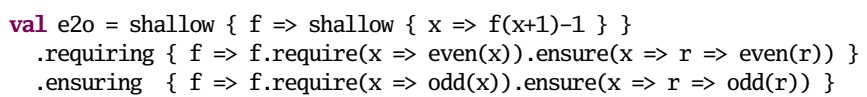

Note the difference between require and requiring: the former takes as argument a predicate of type $\mathrm{A} \Rightarrow$ Rep[Boolean] and asserts it before calling the wrapped function. The latter (requiring) takes as argument a function $\mathrm{A} \Rightarrow \mathrm{A}$ and uses it to merely modify the argument of the wrapped function (adding nested contracts being the prime use case). The same applies to ensures and ensuring.

The example can also be written in shorter form as:

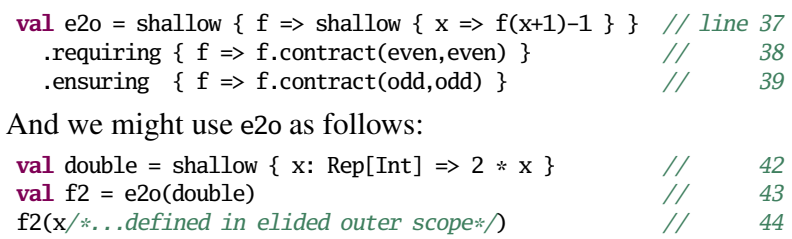

Below is the generated code, with line numbers for every expression. The \#line directives in front of asserts lead the verifier to report contract violations in the correct source locations, according to blame assignment:

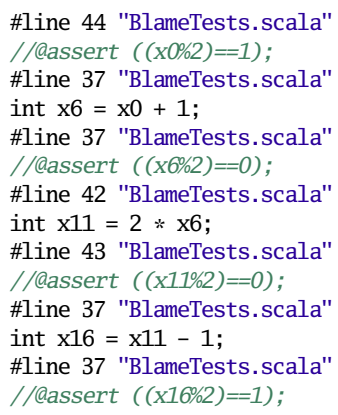

Here, line 37 is the definition of e2o, and lines 42-44 are the three lines of its use. We can see that all parts of the contract, including the higher-order ones, have become first-order assertions. As to blame assignment, we can see that blame correctly switches between the function definition and the call site, blaming the call e2o(double) for any failures of double to return an even number, but blaming the body of e2o for any failures of invoking $f$, and therefore double, with an even number.

Mixing Deep and Shallow Contracts We have looked at contracts for "deep" functions, which are part of the fundef API, and "shallow" functions, which are generation-time macros. 
Since deep functions take Rep arguments, or require an Iso instance, we do not have to deal with passing a shallow function as an argument to a deep function. However, one can pass a deep function as parameter to a shallow function. If the shallow function imposes any contract on its function parameter, then the deep function will be wrapped with code that performs the corresponding checks.

Inductive Predicates Similar to shallow/deep functions on the $\mathrm{C}$ level, we have the same choice for assertions. Either we can abstract at staging time and inline, or we can generate definitions in ACSL.

We already saw ACSL predicates in the context of specifying invariants and deep equality (Figure 2). For deep equality, we saw that we can generate both $\mathrm{C}$ code and a corresponding ACSL predicate from the same Scala source. Here is another small example:

predicate("is_pos", $\{\mathrm{x}$ : Rep[Int] $\Rightarrow \mathrm{x}>0\}$ )

This source generates an ACSL predicate and C code that implements the logic, as ensured by the post-condition:

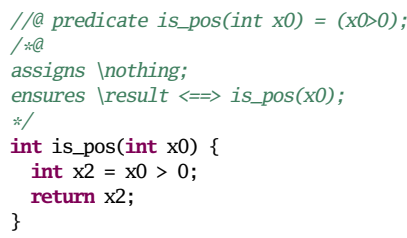

This predicate is_pos can now be used as part of any other spec or code, and will refer to the generated ACSL or C definition, which helps in making generated specifications more readable.

ACSL also supports inductive definitions, as a set of cases with the usual least fixed point semantics. We use this facility for example in our case study on sorting, to specify that the sorted output is a permutation of the input (see Section 5.1).

Properties of Contract Embedding Our generative embedding of contracts achieves much of the expressiveness of state-of-the-art dynamic contract systems [30]. In particular, contracts are composable, higher-order, dependent (they can express dependent relationships between the domain and range), potentially recursive, and they support blame assignment. Our embedding is heavily inspired by soft contract verification [58], but goes beyond it in guaranteeing that all dynamic contract checks are eliminated. Like TreatJS [44], our contracts guarantee non-interference of contracts with normal program code: we generate assertions in a specialized, side-effectfree, language. We control precisely what is necessary to compute only for verification vs for normal program execution. Computations only used in assertions are not emitted as part of the $\mathrm{C}$ code. Thus, adding or deleting contracts has no effect on the computation.

\section{Generative Verification}

Through contracts, we have already seen some of the power of the generative approach extended to verification. Here, we delve deeper into generative patterns for verification that further alleviate the annotation burden.

Type Classes for Invariants Recall from Section 2.2 that the type class Iso controls object representation. An important insight is that type classes can be used not only for shaping code, but also specifications. In particular, they can fill in parts of contracts automatically.

We define a core type class Inv for representation invariants: trait Inv[T] \{ def valid(x:T): Rep[Boolean] \}

which we instantiate for $\mathrm{Vec}[\mathrm{T}]$ as follows:

implicit def vecInv[T:Inv] = invariant [Vec[T] $\{x \Rightarrow$

x.a.valid(0 until x.n) \&\& ( $(0$ until x.n) forall $\{\dot{i} \Rightarrow x(i)$.valid $\})\}$

The helper function invariant acts as default constructor analogous to equality for Eq as in Section 2.1. The given Vec[T] invariant requires that the data pointer a is valid for the full length $n$, and that the nested invariant for $\mathrm{T}$, specified by $\operatorname{Inv}[\mathrm{T}]$, holds for all elements of the vector.

The type classes Inv and Iso co-operate mutually and with fundef so that representation invariants of parameters and return values are enforced in pre- and post-conditions of code functions. Type classes compose and interact transitively as we would expect, and as exemplified by the invariants for $\mathrm{T}$ and $\mathrm{Vec}[\mathrm{T}]$ above. Furthermore, in the generated code for the teaser (Figure 2), the equality predicates require representation invariants in their $\mathrm{C}$ implementations (though not their ACSL definition, deliberately).

Type classes provide a discipline for structuring genericity relying on parametricity by default and explicit functionality as needed - well-suited for composing and specializing specifications, and for automating simple contracts, cutting down on repetitive manual annotations.

Code and Specifications from a Single Source Back in our teaser example (Figures 1 and 2), the definition of equality generates both an ACSL logic predicate and a C implementation. Another example was is_pos in Section 3. Thanks to a single reified IR, we can generate either target from a single IR expression, depending on the context (spec vs code). Thus, we get a big benefit in terms of DRY ("Don't Repeat Yourself"). But how can we be sure that the generated code and the spec actually match? In the generated code in Figure 2, the post-conditions of the eq_vec_functions ensure that the specification is actually implemented.

It is important to stress that we cannot always turn code into specs. For example, side-effecting code has no logic counterpart. Thus we need a way to degrade gracefully. In the case of Eq, the earlier definition from Section 2.1 is refined to:

trait Eq $\{$ def eq(a,b); def eq_spec $(a, b)=e q(a, b)\}$

Method eq_spec is what is used inside specifications. It defaults to the same expression that generates code, but it can be overridden to formulate the spec in another way, if it cannot be directly generated from the code. For example, the code might use caching over the pure eq_spec - a possible pattern to abstract over again.

The expression ( 0 until $\mathrm{n}$ ). forall $\{\mathrm{i} \Rightarrow \ldots\}$ is another interesting case where we capitalize on the benefits of working with an IR instead of generating target code directly. If this expression is used as part of a spec, it will result in an ACSL forall expression:

// $\mid$ forall $i ; 0<=i \& \& i<n=\Rightarrow$...

But if it is used as part of executable code, then an iterative algorithm is generated that enumerates the range and computes the predicate. Another way to look at this is that for predicates that fit the given pattern, we automatically synthesize decision procedures. The take-away here is that a functional programming style lends itself to generating both spec and code from the same source expressions since it can be read both logically and computationally. Deriving Specs from the IR Loop properties and aliasing invariants required for low-level verification (invariant, variant, assigns, separated, ...) are particularly tedious. But in many cases the information we need is already captured as part of internal effect and aliasing information by LMS, and we "just" need to expose it as ACSL annotations.

For most practical purposes, performance-oriented low-level code does not make arbitrary memory accesses but follows some reliable patterns. We introduce a.mutable as a specification internal to LMS-Verify, for a function argument a. The meaning is that mutable function arguments are separate from one another, and separate from immutable data.

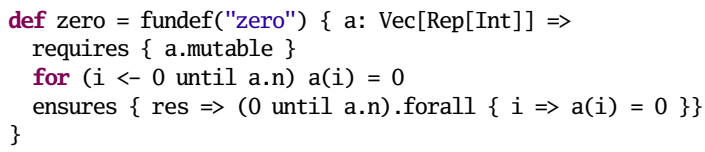


On the IR level, we track modifications, and report any violation of these separation properties as staging-time error. The generated post-conditions ensure that the separation invariants are preserved. The internal implementation is only a slight extension of the preexisting LMS effect system [69], which already keeps track of certain sharing and separation properties for the purpose of optimizations. We also use information from these mutable annotations to generate loop invariants with ACSL assigns annotations.

Domain-Specific Loop Constructs Of course it is unreasonable to expect that we can infer everything we need for all potential use cases. Another powerful pattern to programmatically put invariants in the right places is to introduce higher-level domain-specific abstractions, which combine code, in particular loop patterns, with respective invariants. In the zero snippet above, the expression for ( $i<-0$ until a.n) a(i) $=0$ desugars into Range $(0, a . n)$.foreach $\{i \Rightarrow a(i)=0\}$. The foreach implementation on Range objects can be seen as such a domain-specific abstraction. Internally, it is implemented like this:

def foreach(f: Rep[Int] $\Rightarrow$ Rep[Unit]) $=\{$

var $i=0$; while $(i<n)\{$ loop_invariant $(i>=0 \& \& i<n) ; f(i)\}$ \}

Thus, higher-level abstractions (here, foreach) can automatically insert specific loop invariants, based on domain knowledge (here, about $i$ ). Together with a.mutable and Inv[Vector[Int] ], this loop invariant is enough to validate the postcondition in method zero. We will see another example in Section 5.2.

\section{Case Studies}

We report on four individual case studies:

1. Generic sorting: verify non-trivial properties (e.g. output is inplace sorted permutation of input) and generate specialized versions of a generic library parameterized by element data type, vector data layout, comparator operation. Library generation is an important use-case for generative programming - see Spiral [61] for example.

2. Linear algebra: alleviate the annotation burden by automatically inferring loop and data properties from the intermediate representation and from higher-level knowledge. We showcase scenarios often encountered in DSLs like OptiML [81].

3. Regular expressions: apply the common generative programming pattern of deriving a compiler from an interpreter via staging. We show that we can co-specialize specifications along with the interpreter code.

4. Parser combinators: this case study targets a particularly relevant real-world use case: verifying code that processes untrusted input. Protocol and data format parsing is one of the main sources of security vulnerabilities in the wild. We show that we can write an HTTP parser in a high-level style and generate low-level fast $\mathrm{C}$ code verified to be free of memory and overflow errors.

We present an overview of the required verification effort in Figure 4. For each case study, we list the lines of proofs (LoP) vs lines of code (LoC) in the Scala source, as well as in the generated $\mathrm{C}$. Note that since we are working in a generative setting, we can generate many $\mathrm{C}$ variants, including specifications, from a single Scala source. For some use cases (e.g. sorting) the generator takes more LoC than a single generated target, but the source LoC are amortized over multiple generated versions. In other cases (e.g. HTTP parser) the generated to generator LoC ratio is already high $(\geq 10 \times)$ for a single version. For regular expressions, the generated code size can blow up exponentially as the regular expression grows in backtracking complexitiy. In general, the LoP to LoC ratio is lower than one might expect in the generated code. This is mainly due to the fact that all intermediate expressions are named: each one occupies a separate line in C code, but not in ACSL specs.

\begin{tabular}{|c|c|c|c|c|}
\hline module / instance & LoP & LoC & \# & s. \\
\hline 1. Selection Sort & 41 & 115 & & \\
\hline \multicolumn{5}{|l|}{ verified sorted \& in-place permuted } \\
\hline $\begin{array}{l}\text { ints by } \leq \\
\text { ints by }>\end{array}$ & $\begin{array}{l}88 \\
88\end{array}$ & $\begin{array}{l}26 \\
26\end{array}$ & $\begin{array}{l}70 \\
70\end{array}$ & $\begin{array}{l}7 \\
7\end{array}$ \\
\hline int pairs by first proj. & 116 & 43 & 89 & 11 \\
\hline int pairs by lex. & 130 & 52 & 97 & 20 \\
\hline int vectors by length & 128 & 49 & 109 & 15 \\
\hline $\begin{array}{ll}\text { 2. Linear Algebra } & \text { Scala } \\
\text { verified misc safe \& spec. } & \end{array}$ & 32 & 97 & & \\
\hline matrix,$+ \times$, & 104 & 51 & 101 & 22 \\
\hline member & 23 & 22 & 18 & 2 \\
\hline index where & 26 & 17 & 20 & 3 \\
\hline $\begin{array}{l}\text { 3. Regular Expressions } \\
\text { verified memory safe }\end{array}$ & 3 & 41 & & \\
\hline annotated interpreter & 32 & 35 & 72 & 5 \\
\hline$\wedge \mathrm{a}$ & 4 & 21 & 2 & 1 \\
\hline $\mathrm{C}$ & 10 & 39 & 21 & 11 \\
\hline$a \$$ & 10 & 41 & 24 & 12 \\
\hline$a b . * a b$ & 16 & 155 & 104 & 28 \\
\hline aa* & 16 & 80 & 62 & 13 \\
\hline$a a * b b *$ & 28 & 192 & 160 & 31 \\
\hline$a a * b b * c c *$ & 52 & 416 & 356 & 100 \\
\hline$a a * b b * c c * d d *$ & 100 & 864 & 748 & 320 \\
\hline$a a * b b * c * * d d * e e *$ & 196 & 1760 & 1532 & 1040 \\
\hline $\mathrm{a} a * \mathrm{bb} * \mathrm{cc} * \mathrm{dd} * \mathrm{ee} * \mathrm{ff} *$ & 388 & 3552 & 3100 & 4820 \\
\hline 4. HTTP Parser & 2 & 118 & & \\
\hline staged parser combinators & 5 & 197 & & \\
\hline \multicolumn{5}{|l|}{ verified memory \& overflow safe } \\
\hline without chunking & 95 & 1517 & 276 & 27 \\
\hline with chunking & 133 & 2630 & 2404 & 650 \\
\hline
\end{tabular}

Figure 4. Verification effort for case studies: lines of proof/specification (LoP) \& lines of code (LoC) in Scala code generator and generated $\mathrm{C}$ code, which Frama-C verifies using given number of goals (\#) in given seconds (s.).

\subsection{Generic Sorting}

We write an in-place sort routine - we choose selection sort, though another in-place algorithm such as insertion sort or even quick sort would work as well. This case study is inspired by a hand-written verified sorting function [19], but is considerably more general. Namely, the code is parameterized on an element type $\mathrm{T}$ and on an instance of the type class $0 \mathrm{rd}[\mathrm{T}]$, which defines an ordering, made available through the comparison function cmp:

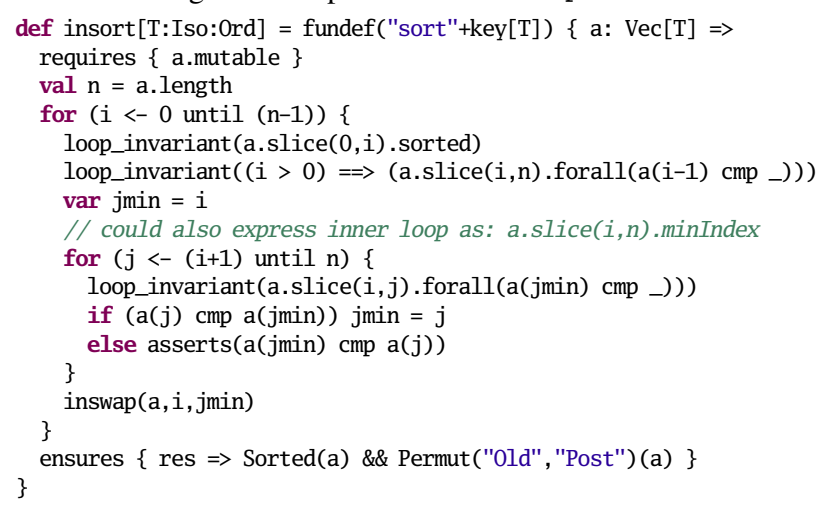

The sort routine operates on a generic Vec, representing an indexed sequence of $n$ elements. We verify that on output, the sequence is a sorted permutation of what it was on input. The a.mutable precondition tracks modifications of vector a. The first post-condition ensures that the sequence is sorted and the second that the sequence on output is a permutation of the sequence on input. The first helper is straightforward to define:

def Sorted[T:Iso:0rd] (a: Vec[T]) = forall $\{i: \operatorname{Rep}[$ Int $] \Rightarrow$

$(0<=i \& \& i<a$.length -1$)=\Rightarrow(a(i) \mathrm{cmp} a(i+1))\}$ 
The second one is a bit more involved. First, note that Permut needs to relate two states of the same data structure. ACSL provides syntax $\backslash a t(a, 01 d)$ and $\backslash a t(a, P o s t)$ for this, which we expose directly in our programming model. We model permutations in the usual way as a a combination of zero or more swap operations. Thus, it is convenient to first define a predicate Swapped:

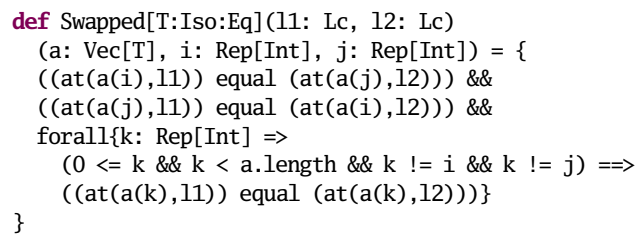

where for equal, we can choose either deep or shallow equality. The parameters 11 and 12 of type Lc range over 0ld,Post, and potentially other modifiers, as in plain ACSL. The helper function inswap already can use Swapped in its post-condition. While Swapped captures the swapping of two specific elements, we actually want to express the property of swapping any two elements for the purpose of defining permutations. We thus add an auxiliary definition Swapped1:

def Swapped1[T:Iso:Eq] (1s: $(\mathrm{Lc}, \mathrm{Lc}))(\mathrm{a}: \operatorname{Vec}[\mathrm{T}])=$

exists $\{i: \operatorname{Rep}[$ Int $] \Rightarrow \operatorname{exists}\{j: \operatorname{Rep}[\operatorname{Int}] \Rightarrow \operatorname{Swapped}(1 s)(a, i, j)\}\}$

In a second step, we define Permut as the reflexive, transitive, closure of Swapped1:

def Permut[T:Iso:Eq] =

reflexiveTransitiveClosure[Vec[T]] (Swapped1, as="Permut")

The combinator reflexiveTransitiveClosure will generate an inductive definition in ACSL. For the instantiation Permut[Rep[Int]], the generated code looks as follows:

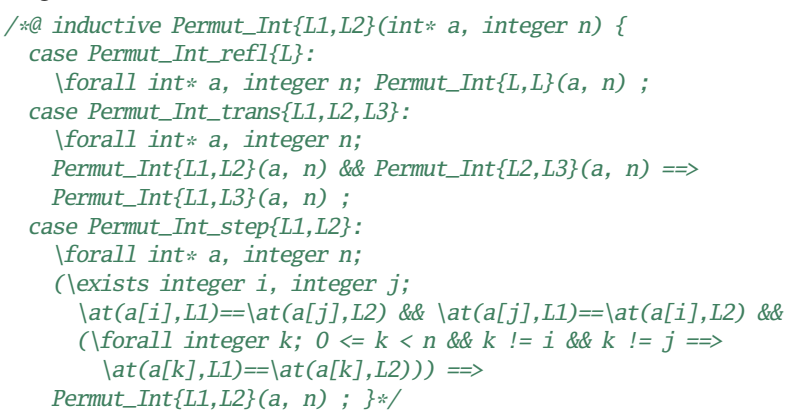

The parameters a and $\mathrm{n}$ correspond to the fields of Vec, according to its Iso definition. The body of the Permut_Int_step case contains the inlined definition of Swapped1 on the left-hand side of the implication $\ldots .=\Rightarrow$ Permut_Int $\{\mathrm{L} 1, \mathrm{~L} 2\}(\mathrm{a}, \mathrm{n})$.

Over the plain version in Frama-C, the first benefit of staging is that we are able to define and use higher-order combinators like reflexiveTransitiveClosure, much like higher-order functions in normal code. The second major benefit is that we can abstract over the datatype and over the comparison function very easily. In particular, the comparator cmp works both as C code and as ACSL specification. From the sort routine, we can derive specialized verified sort functions for any type $\mathrm{T}$ for which we have a comparator. Finally, we can also control the data layout of the sequence itself by varying the Iso type class for Vec and Pointer.

The definition of Ord[T] follows that of Eq[T] seen earlier. What is interesting, although standard type class practice, is that we can define higher-order instances of ord, for example, in order to combine multiple separate orderings into a joint lexicographic ordering. In our generative setting, the Ord instances can also decide whether the comparison will be implemented as a shallow function (i.e. inlined into function insort) or delegate to a generated function.

Since we only verify the generated code, we only verify particular instances of the sort routine, not the generic one (which does not exist as $\mathrm{C}$ code, only in the generator). So what happens if we specialize the sort routine with an unsuitable comparator, for example, one that is not transitive? The generated code will fail to verify. In the generator, we do not have to explicitly require that the comparator be transitive - it will verify when it is, and will not when it isn't. However, we can further explicitly indicate this requirement in the generator, guiding blame assignment to fault the comparator instantiation rather than the sorting routine.

In order to achieve this, we add a method valid to the $\mathrm{Ord}[\mathrm{T}]$ interface, which will define the property of actually being an ordering relation, namely reflexivity, antisymmetry, and transitivity.

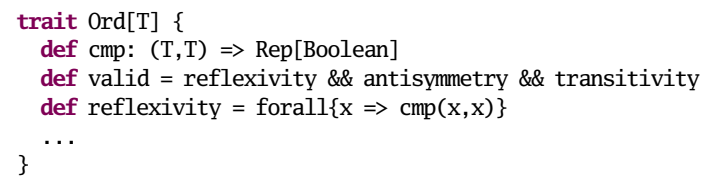

In method insort, we can now add a check ord[T].valid to the precondition, and thus verify the desired properties up front. This will prevent any spurious verification errors in the middle of insort that are due to the provided comparison lacking one of these properties, resulting in more accurate blame assignment.

Finally, this case study illustrates that the overhead of being generic does not translate to much overhead for the programmer in terms of generative verification.

\subsection{Linear Algebra Library}

For this case study, we implement a small linear algebra library with operations on matrices and vectors. First, we define a class Matrix in the same style as the class Vec.

Here is the definition of matrix multiplication, which generates code that fully verifies in Frama-C. The definition is parameterized over the element type $\mathrm{T}$ of the matrix as well as a suitable type class instance specifying a ring over $\mathrm{T}$, an algebraic structure defining (zero,,$+ *$ ).

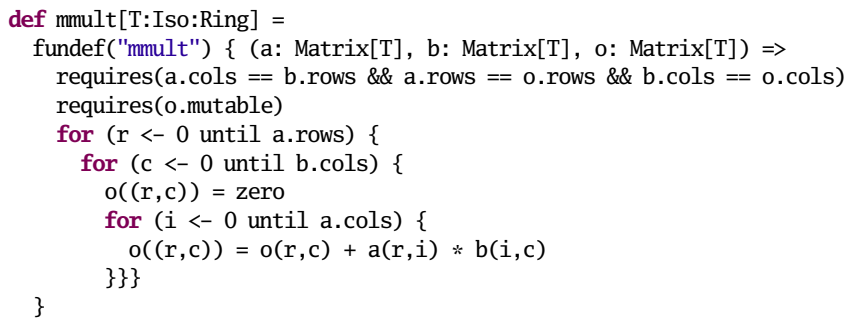

Through Iso[Matrix[T] ], the appropriate data invariants are automatically added as pre- and post- conditions. This is why the remaining explicit pre-condition focuses on the dimension constraints that are specific to the problem at hand. This automation for data invariants also makes it convenient to call these linear algebra routines as one would an external library, without having to worry about annotations.

The framework also automatically infers the loop properties, because they are generic to for-loops and not specific to the problem at hand. For example, here is the ACSL code generated for the first loop for ( $r<-0$ until a.rows), modulo renaming to match source instead of target:

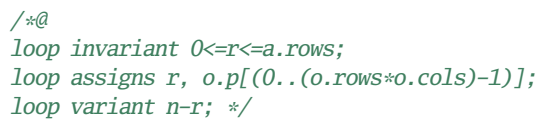

Finally, we can use the host language to structure code and specification logic further. For example, we define pointwise addition and scalar multiplication, delegating to an underlying common staging-time abstraction setFrom: 
def madd[T:Iso:Ring] =

fundef("add") $\{$ (a: Matrix[T], b: Matrix[T], o: Matrix[T]) $\Rightarrow$ o.setFrom2( $\{(\mathrm{ai}: \mathrm{T}, \mathrm{bi}: \mathrm{T}) \Rightarrow \mathrm{ai}+\mathrm{bi}\}, \mathrm{a}, \mathrm{b})\}$

def smult[T:Iso:Ring] =

fundef("smult", \{(a: T, b: Matrix[T], o: Matrix[T]) $\Rightarrow$

o.setFroml $(\{(\mathrm{bi}: \mathrm{T}) \Rightarrow \mathrm{a} * \mathrm{bi}\}, \mathrm{b})$

// easy to prove, thanks to annotations added by setFrom

ensures $\{$ result: Rep[Unit] $\Rightarrow(\mathrm{a}==$ zero $)=\Rightarrow$

(0 until o.rows). forall $\{\mathrm{r} \Rightarrow(0$ until 0. cols $)$. forall $\{\mathrm{c} \Rightarrow$ $o(r, c)==$ zero $\}\}\}\})$

The higher-order staging-time abstraction setFrom in the Matrix[T] class assigns element-wise, mapping the staging-time function argument $f$ over a list of matrices:

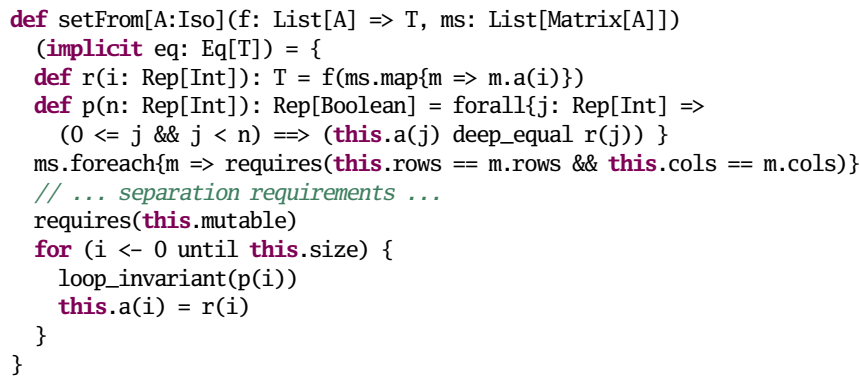

The staging-time function passed in as argument is required to be pure, so it can generate both $\mathrm{C}$ code and ACSL logic. Hence, the staging-time abstraction can also decorate the result with extra specifications, and thanks to these, we can verify further domainspecific properties of scalar multiplication. Also, the staging-time abstraction can add its own pre-condition to require matching dimensions of the argument and output matrices.

In general, we found it a useful pattern to create our own staging-time abstractions that encapsulate verification properties. Here, notice that setFrom does not pass in the position of the element to the (pure) calculation, so we know that each entry is conceptually computed independently. We can also define another abstraction that passes the position (row index, column index) in the matrix, encapsulating the specific separation requirements and properties for this variant.

\subsection{Regular Expression Matcher}

We illustrate that one can often reason once and generically at generation time, producing specialized low-level code along with the annotations to verify it. We take a staged regexp interpreter, which compiles a regular expression to low-level code for matching a string, and by minimally annotating the staged interpreter, produce low-level code that can be verified to be memory-safe.

Our starting point is a simple regular expression matcher [45]. We view it as a tiny interpreter, where the program is the regular expression and the input is the text to match. It is well-known [34] that we can turn an interpreter into a compiler via staging, by making the program (i.e. the regular expression) known and the input (i.e. the text to match) unknown to the generator [76]. Of course, we do not necessarily get an optimizing compiler, but in the case of regexp matchers, we can exploit the connection to finite automata to optimize beyond simple specialization [75, 73].

In this case study, we simply adapt this well-known generative programming pattern of turning an interpreter into a compiler, so that the low-level specialized code verifies to be free of memory errors. It is nice that we can annotate the generic code once, and derive verified code for each regular expression - although we don't verify the generator: we have to verify the generated code with Frama-C for each specialization.

In total, we only need three straightforward annotations in the generator: we simply require that the input text is a valid string, and we annotate each loop with very simple invariants that guide

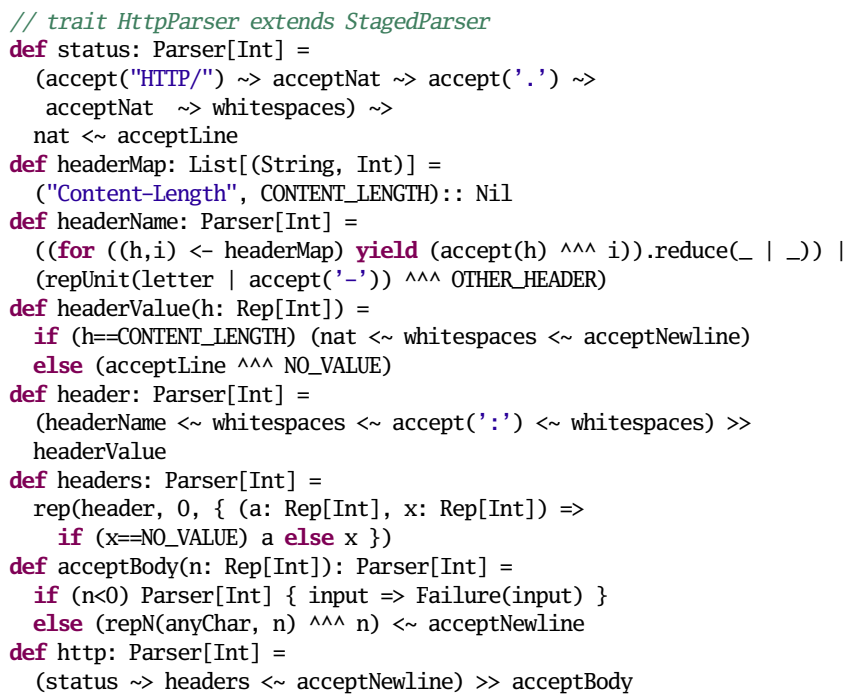

Figure 5. HTTP parser via staged parser combinators in Scala

// trait ChunkedHttpParser extends HttpParser

override def headerMap = super.headerMap :+

("Transfer-Encoding", TRANSFER_ENCODING)

override def headerValue(h: Rep[Int $]$ ) =

if ( $\mathrm{h}==$ TRANSFER_ENCODING)

(accept("chunked") ^^^ CHUNKED) < whitespaces < acceptNewline else super.headerValue( $h$ )

override def acceptBody(n: Rep[Int]): Parser[Int] =

if ( $\mathrm{n}==$ CHUNKED) chunkedBody else super.acceptBody(n)

def hexDigit2Int: Parser[Int] =

digit2Int

(acceptIf(c $\Rightarrow c$ c $>$ unit('a') \&\& c <= unit('f')) $\wedge \wedge$

(c $\Rightarrow 10+(c-$ unit('a')). asInstance0f[Rep[Int]]))

def hex: Parser[Int] = num(hexDigit2Int, 16)

def acceptChunk: Parser[Int] =

(hex < acceptNewline) >> super.acceptBody

def chunkedBody: Parser[Int] =

rep(acceptChunk, 0, \{ (a: Rep[Int], x: Rep[Int]) $\Rightarrow$

if $(a<0) a$

else if (a>Int.MaxValue - $x$ ) OVERFLOW

else $a+x$

\}, overflow0rPos)

Figure 6. HTTP parser extended with chunking

Frama-C to accept that the string is always accessed within its valid range - in the generator, only two source loops are potentially reified into the generated code.

For comparison, we also annotated the original regular expression matcher interpreter, so that Frama-C can verify its memory safety. While straightforward, it is interestingly more work than annotating the generator, because Frama-C needs to ensure that the regular expression is also accessed within range, while of course, in the compiler variant, the regular expression just becomes part of the low-level code. We also had to guide Frama-C with an assertion so that it could see that a recursive call (on a smaller part of the regular expression) was indeed well-founded.

Finally, as expected, the specialized code is usually faster than the original regular expression matcher. For example, on the text acacabc...caba of length 1000000 where ... are all c's, which matches the regular expression $a b . * a b$, the specialized code is about twice as fast as the original interpreter in $\mathrm{C}$.

\subsection{HTTP Parser}

We adapt the work on staged parser combinators [43, 42] to generate $\mathrm{C}$ code instead of Scala code. After this initial port (which amounts to switching the code generation backend of the LMS framework), with minimal additional work, we further ensure that 


\begin{tabular}{|l|l|}
\hline parser & requests per second \\
\hline (baseline) nginx & $(0.94 \pm 0.01) \cdot 10^{6}$ \\
(our) staged verified & $(1.00 \pm 0.01) \cdot 10^{6}$ \\
\hline
\end{tabular}

Figure 7. HTTP parser performance results (higher is better)

the generated $\mathrm{C}$ code verifies completely with Frama-C, guaranteeing the absence of memory and overflow errors. Thus, we obtain a high-performance and secure HTTP parser. While HTTP parsers have caused major vulnerabilities in both Apache and Nginx related to chunk processing [12, 23, 24], we show that we can ensure safety without sacrificing performance nor productivity.

We build our HTTP parser using high-level combinators. Since we can use staging-time abstractions to structure our code without penalty, we build a basic HTTP parser (Figure 5) and extend it to handle chunk processing (Figure 6). The generated code for the HTTP parser is some thousands of lines of low-level C code, a glimpse of which we display in Figure 8.

We show performance results in Figure 7, comparing to the HTTP parser of Nginx [60], which is written in C as well. On a benchmark for parsing HTTP responses and validating the payload length, our approach is competitive, processing one million items per second.

We only need a few changes to go from a working to a verified parser. First, like for the regular expression matcher, we require and ensure that the input remains valid:

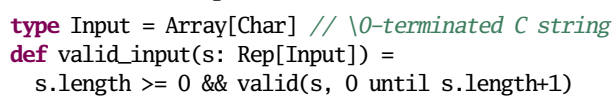

We also need some annotations on loops, but we have very few of those. The combinator rep uses a while loop to repeatedly parse with another combinator $\mathrm{p}$ :

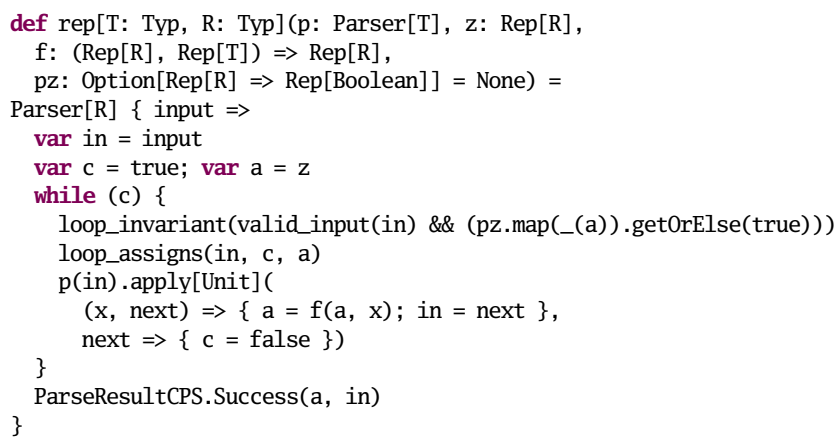

The invariant ensures that the input remains valid, but also, optionally, a custom invariant that depends on the accumulated result.

Finally, we need the parser to behave adequately in case of overflow. In particular, the usual num combinator to parse a number fails to verify, because of possible overflow errors:

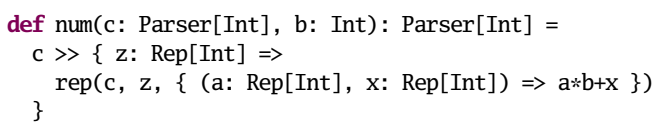

Thus, we overwrite this parser combinator to explicitly handle overflow errors:

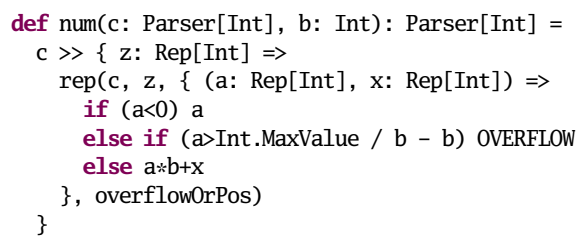

This final step provides the main assurance we were after: the absence of overflows that could be exploited by malicious inputs,

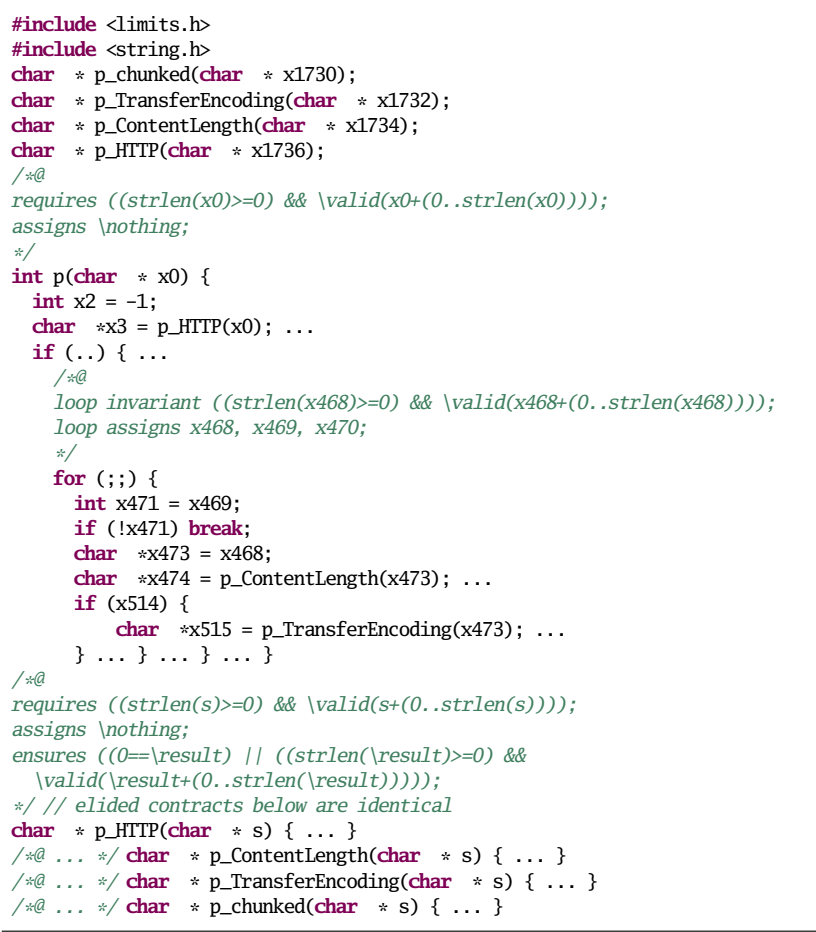

Figure 8. HTTP parser generated $\mathrm{C}$ code

as demonstrated with known vulnerabilities in popular servers like Nginx and Apache [23, 24].

\section{Discussion}

We aim to give a broader perspective on our approach and answer some common questions below.

Why not write code in a low-level language with an advanced type system that ensures memory safety, such as Rust? While Rust [54] seems well on track to become a viable and safer replacement for $\mathrm{C}$ in many situations, we still want the benefits of generative programming and "abstraction without regret": on the one hand performance without giving up on productivity and expressive high-level abstractions, and on the other hand the ability to verify functional correctness via (potentially higher-order) contracts.

Why not use a static analyzer or model checker that has more automation than Frama-C? Wouldn't this remove the need for generating all these annotations? Automatic analysis of $\mathrm{C}$ code is a hard problem, despite some successes. For full static verification of code that may include unbounded loops we are not aware of tools that would improve over Frama-C in a fundamental way, although using other comparable tools like VeriFast [39] would certainly be feasible.

Why not verify high-level code directly? Already, we do not trust the code generator. We would trust a high-level verifier much less: does what is verified correspond to what is generated? Program generators can be thought of as DSLs, with the implied assumption that one needs many of them, and that they can be built up from modules. Thus, the overall system will be in flux and under active development, which makes it appear safer to rely on standard tools for $\mathrm{C}$. There are big teams working behind these tools, industrial best practices, fuzzing attacks on analyzers [22], and so on. Furthermore, because verification is independent, we can afford to take more risks, and be more experimental during code generation.

Why not write everything in Coq and verify the generator? This is a much harder problem, despite much ongoing and very 
promising work in this area $[17,16,91,18,25]$. We settle for proof carrying code / translation validation instead of certified compilers. By generating $\mathrm{C}$ code and using an off-the-shelf verifier we obtain modularity and modular verification. We can interface with other $\mathrm{C}$ code, which can be verified independently. And we also get to use existing, powerful, program generation frameworks (LMS).

Translation validation has a number of practical benefits: we can perform optimizations on the LMS IR, but we are verifying the code after optimization. Any properties expressed as specifications will still hold. This aspect is similar to other recent systems like Cogent $[68,2]$ (which also targets $\mathrm{C}$ ), but it is important to look at the differences, too. Cogent in particular is a stand-alone DSL where "interesting" program properties can be encoded in the type system, e.g. using linear types. The Cogent compiler then uses translation validation to generate verified code. In being a complete language on its own, Cogent does not have any relation to generative programming, which, as mentioned earlier, we crucially want for performance.

How do we ensure that transformations do not remove or tamper with specifications? When checking generic safety properties, such as memory safety, we can do an end-to-end check on the generated code level, and trust the safety claim regardless of the correctness of the generator and its source. For modular verification (where we rely on pre-conditions to ensure safety), we need to be more careful. For functional correctness properties beyond safety, we can either inspect and audit the generated specifications, or, better yet, add a manual layer at the $\mathrm{C}$ level that calls generated $\mathrm{C}$ functions and verifies their properties using externally provided ACSL specifications - again, a benefit of targeting a standard specification language.

\section{Related Work}

The present paper touches aspects of security, verification, formal methods, as well as embedded DSLs and generative programming - all vast spaces of their own. We mention the most important lines of related work below.

Memory Safety Control-flow hijacks based on memory corruption bugs remain the largest attack vector for low-level code. Plainly rewriting $C$ code in safe high-level languages is deemed unrealistic for performance [84], and fully verifying existing C code statically is unrealistic for complexity. This dilemma has lead to an arms race between offensive and defensive techniques [84] based on various kinds of dynamic monitoring (e.g. CFI [1]), which also introduce varying levels of overhead, and are often based on simplifying assumptions that lead to incompatibilities or more sophisticated attacks. Generative programming eliminates the performance drawbacks of high-level languages, but re-introduces security concerns. This paper shows how the two can be reconciled to generate $\mathrm{C}$ code which can be statically verified with reasonable effort.

Parsing as Attack Vector Working with untrusted input requires care. Hence, many vulerabilities arise at this boundary, and are often overlooked. For example, after the celebrated static verification of PolarSSL 1.1.8 [89], a remote code execution vulnerability was found due to a bug in an ASN.1 parser in the X.509 module, which was not part of the verification effort [4, 66]. Exploitable bugs in HTTP parsers have been reported for all major web servers, e.g. Nginx [24] and Apache [23]. A recent paper from the security community makes a case for verified parsers, and in particular mentions parser combinators [12]. In this paper, we used parser generator combinators [43] to build an HTTP parser that is memory and overflow safe, and as fast as Nginx.

Static Verification Beyond "simple" security properties like memory safety, functional correctness requires verification with respect to a specification. There exists many static verification, analysis, and model-checking tools of various degrees of sophistication for a variety of languages, e.g. VeriFast [39] Frama-C [21], Astré [20], Boogie [5], Spec\# [6], Dafny [52], Leon [48], BLAST [36]. Still, building fully verified software remains extremely labor intensive. Notable successes include the seL4 and Verve verified OS kernels [46, 93], the Windows device drivers project [10], and the CompCert verified C compiler [53]. A recent example of translation validation is Cogent $[68,2]$, a DSL for verified systems programming that generates $\mathrm{C}$. The correctness of verification tools is also an important concern [22]. Nonetheless, there are clear signs that formal verification is on the verge of becoming practical for larger classes of software (e.g [57]).

We believe that our work has the potential to accelerate this process by integrating verification with existing generative programming frameworks, which enables pragmatic trade-offs, and in particular allows programmers to transparently use higher-order constructs, which are poorly supported by verification tools, at generation time without leaving a trace in the code that is actually verified. Our work is heavily inspired by research coming out of DARPA's HACMS program [31], in particular Chlipala's Bedrock system [17], related work on verified systems-level software [16, 91, 18], and the DSL-based approaches by Galois and others [28, 37, 62] all pioneering the use of meta-programming for verification.

Contracts The "Design by Contract" programming model has its origin in Eiffel $[55,56]$. Higher-order contracts were introduced by Findler and Felleisen [30], and play a significant role in the Racket ecosystem [29, 26, 79, 88]. Higher-order contract systems have also been proposed for other languages, e.g. JavaScript [44]. The performance overhead of dynamic contract monitoring is known to be large $[80,86,87]$, although clever JIT compilation techniques are a promising avenue to reduce overhead [8].

A key realization is that higher-order contracts only fail at first-order checks. The present paper is greatly inspired by recent work on soft contract verification [58, 59], which uses symbolic execution to unfold higher-order function calls to reach a first-order representation, which can be statically checked. Our approach is based on the same insight, but uses staging-time evaluation to eliminate higher-order functions, instead of symbolic execution. In contrast to soft verification, which works well in many cases, but may leave some dynamic checks in other cases, our meta-language type distinction between normal and staged expressions guarantees the absence of dynamic checks.

Generative Programming Generative or multi-stage programming (MSP, staging for short), as established by Taha and Sheard [85] enables programmers to delay evaluation of certain expressions to a generated stage. MetaOCaml [14] implements a classic staging system based on quasi-quotation. Lightweight Modular Staging (LMS) [72] uses types instead of syntax to identify binding times, and generates an intermediate representation instead of target code [69]. LMS draws inspiration from earlier work such as TaskGraph [9], a C++ framework for program generation and optimization. The idea of representing an embedded language abstractly as methods (finally tagless) is due to Carette et al. [15] and Hofer et al. [38], going back to much earlier work by Reynolds [67]. Compiling embedded DSLs through dynamically generated ASTs was pioneered by Leijen and Meijer [51] and Elliot et al. [27].

A number of high-performance program generators have been built, for example ATLAS [92] (linear algebra), FFTW [33] (discrete fourier transform), and Spiral [63] (general linear transformations). Other systems include PetaBricks [3], CVXgen [35] and Halide [65, 64]. Delite is a compiler framework for embedded DSLs that provides parallelization and heterogeneous code generation on top of LMS [73, 13, 74, 50, 82]. Related work on lowlevel systems oriented programming in high-level languages in- 
cludes [32] and the Singularity operating system [49], the LegoBase database engine [47], and staged parsers for communication protocols [43].

\section{Conclusions}

We make the case that generative programming enables "abstraction without regret" for security and verification as it does for performance. Like for performance, we can use staging-time abstractions and exploit domain-specific and high-level knowledge to keep productivity high while enabling independent verification of the generated C code annotated with ACSL specifications. For example, we generate a low-level fast HTTP parser, verified to be safe of memory and overflow errors, from a high-level source using staged parser combinators with just a handful of lines needed to achieve safety.

Generative programming composes well with verification when the properties to verify match the abstractions used at staging time. In such cases, it is easy to also compose the annotation logic in order to ensure verification. Custom correctness properties require more effort (e.g. sorting), but this effort is not more than what would be required in a non-generative setting, and the generative setting can be further exploited to parameterize use cases over such an effort. Overall, we were pleasantly surprised by (1) how little work is required to go from working to verified (2) how little work is required to go from one instance verified to many (i.e. up to all wanted) instances verified.

By extending generative programming to verification, we hope to enlarge the appeal of the approach beyond high-performance computing to also safety-critical domains. By enabling users in such domains to leverage "abstraction without regret", we hope to accelerate the spreading of formal methods based approaches and static verification to larger classes of software.

\section{Acknowledgments}

The authors thank Viktor Kuncak and Sandrine Blazy for insightful discussions. Manohar Jonnalagedda deserves credit for helping with the HTTP parser case study, which is based on his prior work. This research was supported by NSF through awards 1553471 and 1564207, and by the Swiss FNS through award 200021_166154.

\section{References}

[1] M. Abadi, M. Budiu, Ú. Erlingsson, and J. Ligatti. Control-flow integrity. In ACM Conference on Computer and Communications Security, pages 340-353. ACM, 2005.

[2] S. Amani, A. Hixon, Z. Chen, C. Rizkallah, P. Chubb, L. O'Connor, J. Beeren, Y. Nagashima, J. Lim, T. Sewell, J. Tuong, G. Keller, T. C. Murray, G. Klein, and G. Heiser. Cogent: Verifying high-assurance file system implementations. In ASPLOS, pages 175-188. ACM, 2016.

[3] S. P. Amarasinghe. Petabricks: a language and compiler based on autotuning. In M. Katevenis, M. Martonosi, C. Kozyrakis, and O. Temam, editors, High Performance Embedded Architectures and Compilers, 6th International Conference, HiPEAC 2011, Heraklion, Crete, Greece, January 24-26, 2011. Proceedings, page 3. ACM, 2011.

[4] ARMmbed. PolarSSL security advisory 2014-04, 2015. https: //tls.mbed.org/tech-updates/security-advisories/polarsslsecurity-advisory-2014-04.

[5] M. Barnett, B. E. Chang, R. DeLine, B. Jacobs, and K. R. M. Leino. Boogie: A modular reusable verifier for object-oriented programs. In FMCO, volume 4111 of Lecture Notes in Computer Science, pages 364-387. Springer, 2005.

[6] M. Barnett, M. Fähndrich, K. R. M. Leino, P. Müller, W. Schulte, and $\mathrm{H}$. Venter. Specification and verification: the spec\# experience. Commun. ACM, 54(6):81-91, 2011.
[7] P. Baudin, P. Cuoq, J.-C. Filliâtre, C. Marché, B. Monate, Y. Moy, and V. Prevosto. ACSL: ANSI/ISO C Specification Language, reference manual, version 1.11, 2009-2016. http://frama-c.com/download/acsl.pdf.

[8] S. Bauman, C. F. Bolz, R. Hirschfeld, V. Kirilichev, T. Pape, J. G. Siek, and S. Tobin-Hochstadt. Pycket: a tracing JIT for a functional language. In K. Fisher and J. H. Reppy, editors, Proceedings of the 20th ACM SIGPLAN International Conference on Functional Programming, ICFP 2015, Vancouver, BC, Canada, September 1-3, 2015, pages 22-34. ACM, 2015.

[9] O. Beckmann, A. Houghton, M. R. Mellor, and P. H. J. Kelly. Runtime code generation in $\mathrm{C}++$ as a foundation for domain-specific optimisation. In Domain-Specific Program Generation, pages 291-306, 2003.

[10] J. Berdine, B. Cook, and S. Ishtiaq. Slayer: Memory safety for systems-level code. In CAV, volume 6806 of Lecture Notes in Computer Science, pages 178-183. Springer, 2011.

[11] J. T. Boyland, editor. 29th European Conference on Object-Oriented Programming, ECOOP 2015, July 5-10, 2015, Prague, Czech Republic, volume 37 of LIPIcs. Schloss Dagstuhl - Leibniz-Zentrum fuer Informatik, 2015.

[12] S. Bratus, M. L. Patterson, and A. Shubina. The bugs we have to kill. ; login:: the magazine of USENIX \& SAGE, 40(4):4-10, 2015.

[13] K. J. Brown, A. K. Sujeeth, H. Lee, T. Rompf, H. Chafi, M. Odersky, and K. Olukotun. A heterogeneous parallel framework for domainspecific languages. PACT, 2011.

[14] C. Calcagno, W. Taha, L. Huang, and X. Leroy. Implementing multistage languages using asts, gensym, and reflection. GPCE, pages 5776, 2003.

[15] J. Carette, O. Kiselyov, and C. chieh Shan. Finally tagless, partially evaluated: Tagless staged interpreters for simpler typed languages. $J$. Funct. Program., 19(5):509-543, 2009.

[16] H. Chen, D. Ziegler, T. Chajed, A. Chlipala, M. F. Kaashoek, and N. Zeldovich. Using crash hoare logic for certifying the FSCQ file system. In E. L. Miller and S. Hand, editors, Proceedings of the 25th Symposium on Operating Systems Principles, SOSP 2015, Monterey, CA, USA, October 4-7, 2015, pages 18-37. ACM, 2015.

[17] A. Chlipala. The bedrock structured programming system: combining generative metaprogramming and hoare logic in an extensible program verifier. In G. Morrisett and T. Uustalu, editors, ACM SIGPLAN International Conference on Functional Programming, ICFP'13, Boston, MA, USA - September 25 - 27, 2013, pages 391-402. ACM, 2013.

[18] A. Chlipala. From network interface to multithreaded web applications: A case study in modular program verification. In S. K. Rajamani and D. Walker, editors, Proceedings of the 42nd Annual ACM SIGPLAN-SIGACT Symposium on Principles of Programming Languages, POPL 2015, Mumbai, India, January 15-17, 2015, pages 609-622. ACM, 2015.

[19] U. Costa. Correct sorting with Frama-C and some thoughts on formal methdos, Feb 2011. ulissesaraujo.wordpress.com.

[20] P. Cousot, R. Cousot, J. Feret, L. Mauborgne, A. Miné, D. Monniaux, and X. Rival. The astreé analyzer. In ESOP, volume 3444 of Lecture Notes in Computer Science, pages 21-30. Springer, 2005.

[21] P. Cuoq, F. Kirchner, N. Kosmatov, V. Prevosto, J. Signoles, and B. Yakobowski. Frama-C, pages 233-247. Springer Berlin Heidelberg, Berlin, Heidelberg, 2012.

[22] P. Cuoq, B. Monate, A. Pacalet, V. Prevosto, J. Regehr, B. Yakobowski, and X. Yang. Testing static analyzers with randomly generated programs. In NASA Formal Methods, volume 7226 of Lecture Notes in Computer Science, pages 120-125. Springer, 2012.

[23] CVE. 2002-0392: Apache security advisory. https://cve.mitre.org/cgi-bin/cvename. cgi?name=CVE-20020392.

[24] CVE. 2013-2028: nginx security advisory. https://cve. mitre.org/cgi-bin/cvename. cgi?name=CVE-2013- 
2028 .

[25] B. Delaware, C. Pit-Claudel, J. Gross, and A. Chlipala. Fiat: Deductive synthesis of abstract data types in a proof assistant. In $P O P L$, pages 689-700. ACM, 2015.

[26] C. Dimoulas and M. Felleisen. On contract satisfaction in a higherorder world. ACM Trans. Program. Lang. Syst., 33(5):16, 2011.

[27] C. Elliott, S. Finne, and O. de Moor. Compiling embedded languages J. Funct. Program., 13(3):455-481, 2003.

[28] T. Elliott, L. Pike, S. Winwood, P. C. Hickey, J. Bielman, J. Sharp, E. L. Seidel, and J. Launchbury. Guilt free ivory. In B. Lippmeier, editor, Proceedings of the 8th ACM SIGPLAN Symposium on Haskell, Haskell 2015, Vancouver, BC, Canada, September 3-4, 2015, pages 189-200. ACM, 2015.

[29] M. Felleisen, R. B. Findler, M. Flatt, S. Krishnamurthi, E. Barzilay, J. A. McCarthy, and S. Tobin-Hochstadt. The racket manifesto. In T. Ball, R. Bodík, S. Krishnamurthi, B. S. Lerner, and G. Morrisett, editors, 1st Summit on Advances in Programming Languages, SNAPL 2015, May 3-6, 2015, Asilomar, California, USA, volume 32 of LIPIcs, pages 113-128. Schloss Dagstuhl - Leibniz-Zentrum fuer Informatik, 2015.

[30] R. B. Findler and M. Felleisen. Contracts for higher-order functions In M. Wand and S. L. P. Jones, editors, Proceedings of the Seventh ACM SIGPLAN International Conference on Functional Programming (ICFP '02), Pittsburgh, Pennsylvania, USA, October 4-6, 2002. , pages 48-59. ACM, 2002.

[31] K. Fisher. HACMS: high assurance cyber military systems. In HILT, pages 51-52. ACM, 2012.

[32] D. Frampton, S. M. Blackburn, P. Cheng, R. Garner, D. Grove, J. E. B. Moss, and S. I. Salishev. Demystifying magic: high-level low-level programming. In A. L. Hosking, D. F. Bacon, and O. Krieger, editors, Proceedings of the 5th International Conference on Virtual Execution Environments, VEE 2009, Washington, DC, USA, March 11-13, 2009, pages 81-90. ACM, 2009.

[33] M. Frigo. A fast fourier transform compiler. In PLDI, pages 169-180, 1999.

[34] Y. Futamura. Partial evaluation of computation process, revisited. Higher-Order and Symbolic Computation, 12(4):377-380, 1999.

[35] M. Hanger, T. A. Johansen, G. K. Mykland, and A. Skullestad. Dynamic model predictive control allocation using CVXGEN. In 9th IEEE International Conference on Control and Automation, ICCA 2011, Santiago, Chile, December 19-21, 2011, pages 417-422. IEEE, 2011.

[36] T. A. Henzinger, R. Jhala, and R. Majumdar. The BLAST software verification system. In SPIN, volume 3639 of Lecture Notes in Computer Science, pages 25-26. Springer, 2005.

[37] P. C. Hickey, L. Pike, T. Elliott, J. Bielman, and J. Launchbury. Building embedded systems with embedded dsls. In Jeuring and Chakravarty [40], pages 3-9.

[38] C. Hofer, K. Ostermann, T. Rendel, and A. Moors. Polymorphic embedding of DSLs. In Y. Smaragdakis and J. G. Siek, editors, GPCE, pages 137-148. ACM, 2008.

[39] B. Jacobs, J. Smans, P. Philippaerts, F. Vogels, W. Penninckx, and F. Piessens. Verifast: A powerful, sound, predictable, fast verifier for $\mathrm{C}$ and java. In NASA Formal Methods, volume 6617 of Lecture Notes in Computer Science, pages 41-55. Springer, 2011.

[40] J. Jeuring and M. M. T. Chakravarty, editors. Proceedings of the 19th ACM SIGPLAN international conference on Functional programming, Gothenburg, Sweden, September 1-3, 2014. ACM, 2014.

[41] J. Jeuring and P. Jansson. Polytypic programming. In Advanced Functional Programming, volume 1129 of Lecture Notes in Computer Science, pages 68-114. Springer, 1996.

[42] M. Jonnalagedda. Staged parser combinators and recursion, Sep 2015. manojo.github.io.

[43] M. Jonnalagedda, T. Coppey, S. Stucki, T. Rompf, and M. Odersky.
Staged parser combinators for efficient data processing. In A. P. Black and T. D. Millstein, editors, Proceedings of the 2014 ACM International Conference on Object Oriented Programming Systems Languages \& Applications, OOPSLA 2014, part of SPLASH 2014, Portland, OR, USA, October 20-24, 2014, pages 637-653. ACM, 2014.

[44] M. Keil and P. Thiemann. Treatjs: Higher-order contracts for javascripts. In Boyland [11], pages 28-51.

[45] B. Kernighan and R. Pike. A regular expression matcher. In G. Wilson and A. Oram, editors, Beautiful Code, chapter 1. O'Reilly, 2007.

[46] G. Klein, J. Andronick, K. Elphinstone, T. C. Murray, T. Sewell, R. Kolanski, and G. Heiser. Comprehensive formal verification of an OS microkernel. ACM Trans. Comput. Syst., 32(1):2, 2014.

[47] Y. Klonatos, C. Koch, T. Rompf, and H. Chafi. Building efficient query engines in a high-level language. $P V L D B, 7(10): 853-864$, 2014.

[48] V. Kuncak. Developing verified software using leon. In NFM, volume 9058 of Lecture Notes in Computer Science, pages 12-15. Springer, 2015.

[49] J. R. Larus and G. C. Hunt. The singularity system. Commun. ACM, 53(8):72-79, 2010.

[50] H. Lee, K. J. Brown, A. K. Sujeeth, H. Chafi, T. Rompf, M. Odersky, and K. Olukotun. Implementing domain-specific languages for heterogeneous parallel computing. IEEE Micro, 31(5):42-53, 2011.

[51] D. Leijen and E. Meijer. Domain specific embedded compilers. In DSL, pages 109-122, 1999.

[52] K. R. M. Leino. Dafny: An automatic program verifier for functional correctness. In LPAR (Dakar), volume 6355 of Lecture Notes in Computer Science, pages 348-370. Springer, 2010.

[53] X. Leroy. Formal verification of a realistic compiler. Commun. ACM, 52(7):107-115, 2009.

[54] N. D. Matsakis and F. S. Klock, II. The Rust language. Ada Lett., 34(3):103-104, Oct. 2014

[55] B. Meyer. Eiffel: A language and environment for software engineering. Journal of Systems and Software, 8(3):199-246, 1988.

[56] B. Meyer. Eiffel as a framework for verification. In VSTTE, volume 4171 of Lecture Notes in Computer Science, pages 301-307. Springer, 2005.

[57] C. Newcombe, T. Rath, F. Zhang, B. Munteanu, M. Brooker, and M. Deardeuff. How amazon web services uses formal methods. Communications of the ACM, 58(4):66-73, 2015.

[58] P. C. Nguyen, S. Tobin-Hochstadt, and D. V. Horn. Soft contract verification. In Jeuring and Chakravarty [40], pages 139-152.

[59] P. C. Nguyen, S. Tobin-Hochstadt, and D. V. Horn. Higher-order symbolic execution for contract verification and refutation. 2015.

[60] nodejs \& nginx. HTTP parser. https://github.com/nodejs/http-parser.

[61] G. Ofenbeck, T. Rompf, A. Stojanov, M. Odersky, and M. Püschel. Spiral in scala: towards the systematic construction of generators for performance libraries. In J. Järvi and C. Kästner, editors, Generative Programming: Concepts and Experiences, GPCE'13, Indianapolis, IN, USA - October 27 - 28, 2013, pages 125-134. ACM, 2013.

[62] L. Pike, P. C. Hickey, J. Bielman, T. Elliott, T. DuBuisson, and J. Launchbury. Programming languages for high-assurance autonomous vehicles: extended abstract. In N. A. Danielsson and B. Jacobs, editors, Proceedings of the 2014 ACM SIGPLAN Workshop on Programming Languages meets Program Verification, PLPV 2014, January 21, 2014, San Diego, California, USA, Co-located with POPL '14, pages 1-2. ACM, 2014.

[63] M. Püschel, J. M. F. Moura, B. Singer, J. Xiong, J. Johnson, D. A. Padua, M. M. Veloso, and R. W. Johnson. Spiral: A generator for platform-adapted libraries of signal processing alogorithms. IJHPCA, 18(1):21-45, 2004. 
[64] J. Ragan-Kelley, A. Adams, S. Paris, M. Levoy, S. P. Amarasinghe, and F. Durand. Decoupling algorithms from schedules for easy optimization of image processing pipelines. ACM Trans. Graph., 31(4):32, 2012.

[65] J. Ragan-Kelley, C. Barnes, A. Adams, S. Paris, F. Durand, and S. P. Amarasinghe. Halide: a language and compiler for optimizing parallelism, locality, and recomputation in image processing pipelines. In H. Boehm and C. Flanagan, editors, ACM SIGPLAN Conference on Programming Language Design and Implementation, PLDI '13, Seattle, WA, USA, June 16-19, 2013, pages 519-530. ACM, 2013.

[66] J. Regehr. Comments on a formal verification of PolarSSL, 2015. http://blog.regehr.org/archives/1261.

[67] J. Reynolds. User-defined types and procedural data structures as complementary approaches to data abstraction. 1975.

[68] C. Rizkallah, J. Lim, Y. Nagashima, T. Sewell, Z. Chen, L. O'Connor, T. C. Murray, G. Keller, and G. Klein. A framework for the automatic formal verification of refinement from cogent to C. In ITP, volume 9807 of Lecture Notes in Computer Science, pages 323-340. Springer, 2016.

[69] T. Rompf. Lightweight Modular Staging and Embedded Compilers: Abstraction Without Regret for High-Level High-Performance Programming. PhD thesis, EPFL, 2012.

[70] T. Rompf, N. Amin, A. Moors, P. Haller, and M. Odersky. Scalavirtualized: Linguistic reuse for deep embeddings. Higher-Order and Symbolic Computation (Special issue for PEPM'12).

[71] T. Rompf, K. J. Brown, H. Lee, A. K. Sujeeth, M. Jonnalagedda, N. Amin, G. Ofenbeck, A. Stojanov, Y. Klonatos, M. Dashti, C. Koch, M. Püschel, and K. Olukotun. Go meta! A case for generative programming and dsls in performance critical systems. In SNAPL, 2015.

[72] T. Rompf and M. Odersky. Lightweight modular staging: a pragmatic approach to runtime code generation and compiled dsls. Commun. ACM, 55(6):121-130, 2012.

[73] T. Rompf, A. K. Sujeeth, N. Amin, K. Brown, V. Jovanovic, H. Lee, M. Jonnalagedda, K. Olukotun, and M. Odersky. Optimizing data structures in high-level programs. POPL, 2013.

[74] T. Rompf, A. K. Sujeeth, H. Lee, K. J. Brown, H. Chafi, M. Odersky, and $\mathrm{K}$. Olukotun. Building-blocks for performance oriented dsls. DSL, 2011.

[75] Scala-LMS. Tutorial: Automata-based regex matcher. http://scala-lms.github.io/tutorials/automata.html.

[76] Scala-LMS. Tutorial: From interpreter to compiler. http://scala-lms.github.io/tutorials/regex.html.

[77] A. Slesarenko, A. Filippov, and A. Romanov. First-class isomorphic specialization by staged evaluation. In WGP, pages 35-46. ACM, 2014.

[78] C. Strachey. Fundamental concepts in programming languages. Higher-Order and Symbolic Computation, 13(1/2):11-49, 2000.

[79] T. S. Strickland, C. Dimoulas, A. Takikawa, and M. Felleisen. Contracts for first-class classes. ACM Trans. Program. Lang. Syst.,
35(3):11, 2013.

[80] T. S. Strickland, S. Tobin-Hochstadt, R. B. Findler, and M. Flatt. Chaperones and impersonators: run-time support for reasonable interposition. In G. T. Leavens and M. B. Dwyer, editors, Proceedings of the 27th Annual ACM SIGPLAN Conference on Object-Oriented Programming, Systems, Languages, and Applications, OOPSLA 2012, part of SPLASH 2012, Tucson, AZ, USA, October 21-25, 2012, pages 943-962. ACM, 2012.

[81] A. K. Sujeeth, H. Lee, K. J. Brown, T. Rompf, M. Wu, A. R. Atreya, M. Odersky, and K. Olukotun. OptiML: an implicitly parallel domainspecific language for machine learning. In Proceedings of the 28th International Conference on Machine Learning, ICML, 2011.

[82] A. K. Sujeeth, T. Rompf, K. J. Brown, H. Lee, H. Chafi, V. Popic, M. Wu, A. Prokopec, V. Jovanovic, M. Odersky, and K. Olukotun. Composition and reuse with compiled domain-specific languages. In ECOOP, 2013.

[83] J. Svenningsson and E. Axelsson. Combining deep and shallow embedding for EDSL. In TFP, 2012.

[84] L. Szekeres, M. Payer, T. Wei, and D. Song. Sok: Eternal war in memory. In IEEE Symposium on Security and Privacy, pages 48-62. IEEE Computer Society, 2013.

[85] W. Taha and T. Sheard. Metaml and multi-stage programming with explicit annotations. Theor. Comput. Sci., 248(1-2):211-242, 2000.

[86] A. Takikawa, D. Feltey, E. Dean, M. Flatt, R. B. Findler, S. TobinHochstadt, and M. Felleisen. Towards practical gradual typing. In Boyland [11], pages 4-27.

[87] A. Takikawa, D. Feltey, B. Greenman, M. New, J. Vitek, and M. Felleisen. Is sound gradual typing dead? In POPL, 2016.

[88] S. Tobin-Hochstadt and M. Felleisen. The design and implementation of typed scheme. In G. C. Necula and P. Wadler, editors, Proceedings of the 35th ACM SIGPLAN-SIGACT Symposium on Principles of Programming Languages, POPL 2008, San Francisco, California, USA, January 7-12, 2008, pages 395-406. ACM, 2008.

[89] TrustInSoft. PolarSSL 1.1.8 verification kit, 2015. http://trust-in-soft.com/polarSSL_demo.pdf.

[90] P. Wadler and S. Blott. How to make ad-hoc polymorphism less adhoc. In POPL, pages 60-76, 1989.

[91] X. Wang, D. Lazar, N. Zeldovich, A. Chlipala, and Z. Tatlock. Jitk: A trustworthy in-kernel interpreter infrastructure. In J. Flinn and H. Levy, editors, 11th USENIX Symposium on Operating Systems Design and Implementation, OSDI '14, Broomfield, CO, USA, October 6-8, 2014., pages 33-47. USENIX Association, 2014.

[92] R. C. Whaley, A. Petitet, and J. Dongarra. Automated empirical optimizations of software and the ATLAS project. Parallel Computing, 27(1-2):3-35, 2001.

[93] J. Yang and C. Hawblitzel. Safe to the last instruction: automated verification of a type-safe operating system. Commun. ACM, 54(12):123131, 2011. 